\title{
Perron-Frobenius operators and the Klein-Gordon equation
}

\author{
Francisco Canto-Martín, Håkan Hedenmalm, and Alfonso Montes-Rodríguez
}

\begin{abstract}
For a smooth curve $\Gamma$ and a set $\Lambda$ in the plane $\mathbb{R}^{2}$, let $\operatorname{AC}(\Gamma ; \Lambda)$ be the space of finite Borel measures in the plane supported on $\Gamma$, absolutely continuous with respect to the arc length and whose Fourier transform vanishes on $\Lambda$. Following [12, we say that $(\Gamma, \Lambda)$ is a Heisenberg uniqueness pair if $\mathrm{AC}(\Gamma ; \Lambda)=\{0\}$. In the context of a hyperbola $\Gamma$, the study of Heisenberg uniqueness pairs is the same as looking for uniqueness sets $\Lambda$ of a collection of solutions to the Klein-Gordon equation. In this work, we mainly address the issue of finding the dimension of $\operatorname{AC}(\Gamma ; \Lambda)$ when it is nonzero. We will fix the curve $\Gamma$ to be the hyperbola $x_{1} x_{2}=1$, and the set $\Lambda=\Lambda_{\alpha, \beta}$ to be the lattice-cross

$$
\Lambda_{\alpha, \beta}=(\alpha \mathbb{Z} \times\{0\}) \cup(\{0\} \times \beta \mathbb{Z}),
$$

where $\alpha, \beta$ are positive reals. We will also consider $\Gamma_{+}$, the branch of $x_{1} x_{2}=1$ where $x_{1}>0$. In 12], it is shown that $\operatorname{AC}\left(\Gamma ; \Lambda_{\alpha, \beta}\right)=\{0\}$ if and only if $\alpha \beta \leq 1$. Here, we show that for $\alpha \beta>1$, we get a rather drastic "phase transition": $\operatorname{AC}\left(\Gamma ; \Lambda_{\alpha, \beta}\right)$ is infinite-dimensional whenever $\alpha \beta>1$. It is shown in 13. that $\mathrm{AC}\left(\Gamma_{+} ; \Lambda_{\alpha, \beta}\right)=\{0\}$ if and only if $\alpha \beta<4$. Moreover, at the edge $\alpha \beta=4$, the behavior is more exotic: the space $\mathrm{AC}\left(\Gamma_{+} ; \Lambda_{\alpha, \beta}\right)$ is one-dimensional. Here, we show that the dimension of $\mathrm{AC}\left(\Gamma_{+} ; \Lambda_{\alpha, \beta}\right)$ is infinite whenever $\alpha \beta>4$. Dynamical systems, and more specifically Perron-Frobenius operators, will play a prominent role in the presentation.
\end{abstract}

\section{Introduction}

\subsection{Background: the Heisenberg uncertainty principle}

The Heisenberg uncertainty principle asserts that it is not possible to have completely accurate information about the position and the momentum of a particle at the same time. If $\psi$ is the spatial wave-function, which describes the position of the particle in question, and it is known that $\psi$ is concentrated to a small region, then the deviation of the momentum wave-function of $\psi$ from its mean must be large. The momentum wave-function is essentially the Fourier transform of the spatial wave-function. So, we may consider the Heisenberg uncertainty principle as the mathematical statement that a function and its Fourier transform cannot both be too concentrated simultaneously; cf. [2], [10], and [11].

1991 Mathematics Subject Classification. Primary 42B10, 42A10, 58F11; Secondary 11K50, 31B35, 43A15, 81Q05. Key words and phrases. Trigonometric system, inversion, composition operator, Klein-Gordon equation, ergodic theory.

Research partially supported by Plan Nacional ref MTM2009-09501. Research of the first author partially supported by Ministerio de Educación (FPU). Research of the second author partially supported by the Göran Gustafsson Foundation (KVA) and by Vetenskapsrådet (VR). Research of the third author partially supported by Junta de Andalucía ref FQM260. 


\subsection{Heisenberg uniqueness pairs}

Let $\Gamma$ be a finite disjoint union of smooth curves in the plane and $\Lambda$ a subset of the plane. Let $\mathrm{AC}(\Gamma ; \Lambda)$ be the space of bounded Borel measures $\mu$ in the plane supported on $\Gamma$, absolutely continuous with respect to arc length and whose Fourier transform

$$
\widehat{\mu}\left(x_{1}, x_{2}\right)=\int_{\Gamma} \mathrm{e}^{\mathrm{i} \pi\left(x_{1} y_{1}+x_{2} y_{2}\right)} \mathrm{d} \mu\left(y_{1}, y_{2}\right), \quad\left(x_{1}, x_{2}\right) \in \mathbb{R}^{2},
$$

vanishes on $\Lambda$. Following [12, we say that $(\Gamma, \Lambda)$ is a Heisenberg uniqueness pair if $\operatorname{AC}(\Gamma ; \Lambda)=\{0\}$. When $\Gamma$ is an algebraic curve, that is, the zero locus of a polynomial $p$ in two variables with real coefficients, the requirement that the support of $\mu$ be contained in $\Gamma$ means that $\widehat{\mu}$ solves the partial differential equation

$$
p\left(\frac{\partial_{x_{1}}}{\pi i}, \frac{\partial_{x_{2}}}{\pi i}\right) \widehat{\mu}=0
$$

So, there is a natural interplay between the Heisenberg uniqueness pairs and the theory of partial differential equations (PDE), cf. [12. The most natural examples appear we consider quadratic polynomials $p$ corresponding to the standard conic sections: the line, two parallel lines, two crossing lines, the hyperbola, the ellipse, and the parabola. The natural invariance of Heisenberg uniqueness pairs under affine transformations of the plane allows us to reduce to the canonical models for these curves (cf. [12]). The case when $\Gamma$ is either one line or the union of two parallel lines was solved completely for general $\Lambda \subset \mathbb{R}^{2}$ in [12. In this direction, Blasi-Babot has solved particular cases when $\Gamma$ is the union of three parallel lines, see [4. The case when $\Gamma$ is a circle (which also covers the ellipse case after an affine mapping) was recently studied independently by Lev and by Sjölin in [25], 20], where e.g., circles and unions of straight lines are considered as sets $\Lambda$. Also subsets of the unions of straight lines were considered, and a connection with the Beurling-Malliavin theory was made. Very little seems to be known when $\Gamma$ is a parabola or two intersecting lines.

\subsection{Heisenberg uniqueness pairs for the hyperbola}

The case of the hyperbola $\Gamma: x_{1} x_{2}=1$ and the lattice-cross

$$
\Lambda_{\alpha, \beta}=(\alpha \mathbb{Z} \times\{0\}) \cup(\{0\} \times \beta \mathbb{Z}),
$$

for given positive reals $\alpha, \beta$ was considered in [12, where we the following result was obtained.

Theorem A (Hedenmalm, Montes-Rodríguez). Let $\Gamma$ be the hyperbola $x_{1} x_{2}=1$. Then $(\Gamma, \Lambda)$ is a Heisenberg uniqueness pair if and only if $\alpha \beta \leq 1$.

When one of the branches of the hyperbola is considered, the critical density changes, see [13.

Theorem B (Hedenmalm, Montes-Rodríguez). Let $\Gamma_{+}$be the branch of the hyperbola $x_{1} x_{2}=1$ where $x_{1}>0$. Then $\operatorname{AC}\left(\Gamma_{+} ; \Lambda_{\alpha, \beta}\right)=\{0\}$ if and only if $\alpha \beta<4$. Moreover, when $\alpha \beta=4, \operatorname{AC}\left(\Gamma_{+} ; \Lambda_{\alpha, \beta}\right)$ is one-dimensional.

For sub-critical density of the lattice-cross, we have the following two theorems, corresponding to the hyperbola and a branch of the hyperbola.

Theorem 1.1. Let $\Gamma$ be the hyperbola $x_{1} x_{2}=1$. Then $\mathrm{AC}\left(\Gamma ; \Lambda_{\alpha, \beta}\right)$ is infinite-dimensional for $\alpha \beta>1$.

Theorem 1.2. Let $\Gamma_{+}$be the branch of the hyperbola $x_{1} x_{2}=1$ with $x_{1}>0$. Then the space $\mathrm{AC}\left(\Gamma_{+} ; \Lambda_{\alpha, \beta}\right)$ is infinite-dimensional for $\alpha \beta>4$.

Although the proofs of Theorem 1.1 and 1.2 share a certain degree of parallelism, the proof of Theorem 1.1] is more delicate than that of Theorem[1.2. Mainly, the difference is that at the edge $\alpha \beta=4$, the Perron-Frobenius operator which appears in the context of of Theorem 1.2, induced by the classical Gauss map, has a spectral gap [acting on the space of functions of bounded variation], while for the Perron-Frobenius operator associated to the edge case $\alpha \beta=1$ in the context of 
Theorem 1.1 does not have such a spectral gap; this is so because the Gauss-type transformation which defines the Perron-Frobenius operator has an indifferent fixed point.

The basic invariance properties of Heisenberg uniqueness pairs allow us to take $\alpha=1$ and we may appeal to duality and reformulate Theorem $\mathrm{A}$ as follows (cf. [12]).

Theorem $\mathbf{A}^{\prime}$. Let $\mathcal{M}_{\beta}$ be the linear subspace of $L^{\infty}(\mathbb{R})$ spanned by the functions $x \mapsto \mathrm{e}^{\mathrm{i} m \pi x}$ and $x \mapsto \mathrm{e}^{\mathrm{i} n \pi \beta / x}$, where $m, n$ range over the integers and $\beta$ is a fixed positive real. Then $\mathcal{M}_{\beta}$ is weak-star dense in $L^{\infty}(\mathbb{R})$ if and only if $\beta \leq 1$.

The analogous reformulation of Theorem 1.1 runs as follows:

Theorem 1.3. Let $\mathcal{M}_{\beta}$ be the linear subspace of $L^{\infty}(\mathbb{R})$ spanned by the functions $x \mapsto \mathrm{e}^{\mathrm{i} m \pi x}$ and $x \mapsto \mathrm{e}^{\mathrm{i} n \pi \beta / x}$, where $m, n$ range over the integers. Then the weak-star closure of $\mathcal{M}_{\beta}$ in $L^{\infty}(\mathbb{R})$ has infinite codimension in $L^{\infty}(\mathbb{R})$ for $\beta>1$.

If we instead take $\alpha=2$, we may reformulate Theorems $\mathrm{B}$ and 1.2 as follows.

Theorem $\mathbf{B}^{\prime}$. Let $\mathcal{N}_{\beta}$ be the linear subspace of $L^{\infty}\left(\mathbb{R}_{+}\right)$spanned by the functions $x \mapsto \mathrm{e}^{\mathrm{i} 2 m \pi x}$ and $x \mapsto \mathrm{e}^{\mathrm{i} n \pi \beta / x}$, where $m, n$ range over the integers and $\beta$ is a fixed positive real. Then $\mathcal{N}_{\beta}$ is weakstar dense in $L^{\infty}\left(\mathbb{R}_{+}\right)$if and only if $\beta<2$. Moreover, the weak-star closure of $\mathcal{N}_{\beta}$ in $L^{\infty}\left(\mathbb{R}_{+}\right)$has codimension 1 in $L^{\infty}\left(\mathbb{R}_{+}\right)$for $\beta=2$.

Theorem 1.4. Let $\mathcal{N}_{\beta}$ be the linear subspace of $L^{\infty}\left(\mathbb{R}_{+}\right)$spanned by the functions $x \mapsto \mathrm{e}^{\mathrm{i} 2 m \pi x}$ and $x \mapsto \mathrm{e}^{\mathrm{i} n \pi \beta / x}$, where $m, n$ range over the integers and $\beta$ is a fixed positive real. Then the weak-star closure of $\mathcal{N}_{\beta}$ has infinite codimension in $L^{\infty}\left(\mathbb{R}_{+}\right)$for $\beta>2$.

By general Functional Analysis, the codimension of the weak-star closure of $\mathcal{M}_{\beta}$ equals the dimension of its pre-annihilator space

$$
\mathcal{M}_{\beta}^{\perp}=\left\{f \in L^{1}(\mathbb{R}): \int_{\mathbb{R}} f(x) \mathrm{e}^{\mathrm{i} n \pi x} \mathrm{~d} x=\int_{\mathbb{R}} f(x) \mathrm{e}^{\mathrm{i} n \pi \beta / x} \mathrm{~d} x=0 \text { for all } n \in \mathbb{Z}\right\} .
$$

Likewise, the codimension of the weak-star closure of $\mathcal{N}_{\beta}$ equals the dimension of its pre-annihilator space

$$
\mathcal{N}_{\beta}^{\perp}=\left\{f \in L^{1}\left(\mathbb{R}_{+}\right): \int_{\mathbb{R}_{+}} f(x) \mathrm{e}^{\mathrm{i} 2 n \pi x} \mathrm{~d} x=\int_{\mathbb{R}_{+}} f(x) \mathrm{e}^{\mathrm{i} n \pi \beta / x} \mathrm{~d} x=0 \text { for all } n \in \mathbb{Z}\right\} .
$$

If $f \in \mathcal{N}_{\beta}^{\perp}$, then it is easy to see that the function $g(x)=f\left(\frac{1}{2} x\right)$, extended to vanish along the negative semi-axis $\mathbb{R}_{-}$, belongs to $\mathcal{M}_{2 \beta}^{\perp}$. So, Theorems 1.3 and 1.4 show that there are elements in $\mathcal{M}_{\beta}^{\perp}$ with support on the positive semi-axis precisely when $\beta \geq 4$.

Corollary 1.5. In the pre-annihilator $\mathcal{M}_{\beta}^{\perp}$ there exists a non-trivial element that vanishes on $\mathbb{R}_{-}$if and only if $\beta \geq 4$. Moreover, if $\beta=4$, there is only a one-dimensional subspace of such elements, while if $\beta>4$, there is an infinite-dimensional subspace with this property.

Remark 1.6. In the context of Theorems 1.3 and 1.4, we actually construct rather concrete infinitedimensional subspaces of $\mathcal{M}_{\beta}^{\perp}$ and $\mathcal{N}_{\beta}^{\perp}$, respectively; cf. Theorems 8.2 and 8.6 .

\subsection{Discussion about harmonic extension and the codimension problem}

If $\Gamma$ is the hyperbola $x_{1} x_{2}=1$, then for $\beta>1$ the bounded harmonic extensions to the upper half-plane of the functions $x \mapsto \mathrm{e}^{\mathrm{i} m \pi x}$ and $x \mapsto \mathrm{e}^{\mathrm{i} n \pi \beta / x}$, where $m, n$ range over the integers $\mathbb{Z}$, fails to separate all the points of the upper half-plane $\mathbb{C}_{+}:=\{z \in \mathbb{C}: \operatorname{Im} z>0\}$. Indeed, if we consider

$$
z_{1}:=m+\mathrm{i} \sqrt{\frac{\beta}{m n}-1}, \quad z_{2}:=-m+i \sqrt{\frac{\beta}{m n}-1}, \quad \text { where } \quad m, n \in \mathbb{Z}_{+}, \quad m n<\beta,
$$

then, $f\left(z_{1}\right)=f\left(z_{2}\right)$ for every $f \in \mathcal{M}_{\beta}$, so that the differences of Poisson kernels $P_{z_{z}}-P_{z_{2}}$ are elements in the pre-annihilator space $\mathcal{M}_{\beta}^{\perp}$. If we use the Cauchy kernel in place of the Poisson kernel here we also obtain elements of the pre-annihilator. But there are only finitely many combinations of $m, n \in \mathbb{Z}_{+}$with $m n<\beta$, which corresponds to finitely many differences of Poisson 
or Cauchy kernels. This would lead us to suspect that that the pre-annihilator $\mathcal{M}_{\beta}^{\perp}$ might be finite-dimensional. Theorem 1.3 shows that this is far from being true.

\subsection{Structure of the paper}

In Section 2, we take a closer look at the link between Theorem 1.1 and the Klein-Gordon and Dirac equations. In order to make the paper accessible to a wider audience, we present in Section 3 the elementary aspects of the theory of dynamical systems and the standard notation for Perron-Frobenius operators needed here. In Section 4 we show how the theory of Perron-Frobenius operators is the natural tool to analyze Heisenberg uniqueness pairs for the hyperbola $\Gamma$ and for one of its branches $\Gamma_{+}$. In particular, the famous Gauss-Kuzmin-Wirsing operator corresponds to critical density case for $\Gamma_{+}$. In Section [5] we state some more involved results of the theory of Perron-Frobenius operators which are needed later on. In Section 6, we study the structure of the pre-annihilator space $\mathcal{M}_{\beta}^{\perp}$ associated with $\Gamma$. In Section $[7$ we show the existence and uniqueness of absolutely continuous invariant measure for certain transformations acting on the interval $[-1,1]$. This is the key point in the proof of Theorem 1.3, presented in Section 8 , We end Section 8 by sketching the proof of Theorem 1.4, which turns out to be much simpler than that of Theorem 1.3. Finally, in Section 9, we apply our results to a problem involving the linear span of powers of two atomic singular inner functions in the Hardy space of the unit disk. In conclusion, we can say that the study of Heisenberg uniqueness pairs related to the Klein-Gordon equation leads to new and interesting problems involving Perron-Frobenius operators.

\subsection{Acknowledgements}

We thank Michael Benedicks for enlightening discussions on Perron-Frobenius operators.

\section{Further motivation. The Klein-Gordon and Dirac equations}

\subsection{The Dirac equation in three spatial dimensions}

In quantum mechanics the evolution of the position wave-function $\psi$ associated to a physical system can be modelled by certain partial differential equations (PDE). According to the theory of spin, in the general setting, $\psi$ has four components,

$$
\psi=\left(\psi_{1}, \psi_{2}, \psi_{3}, \psi_{4}\right)
$$

which should be thought of as written in column form, where each $\psi_{j}=\psi_{j}\left(t, x_{1}, x_{2}, x_{3}\right)$ is a mapping between an open set in $\mathbb{R}^{4}$ and a prescribed Hilbert space. Thus, these PDE's have to be understood as a system of equations for four separate wave-functions. The necessity of working with multiple-component wave-functions was pointed out by Pauli in order to understand the intrinsic angular momentum (spin) of atoms. There is not a general equation whose solutions reflect faithfully the evolution of a given system from a relativistic point of view. Depending on the features of the system one must choose one or another type of equation. For instance, for a relativistic spin-0 particle with rest-mass $m_{0}$ we have the Klein-Gordon equation. Written in natural units it takes the form,

$$
\left(\partial_{t}^{2}-\partial_{x_{1}}^{2}-\partial_{x_{2}}^{2}-\partial_{x_{3}}^{2}+m_{0}^{2}\right) \psi=0 .
$$

Another example, perhaps the most important in this context, is the Dirac equation. It is used to describe the wave-function of the electron, although it remains valid when applied to a general relativistic spin- $\frac{1}{2}$ particle. In natural units it takes the form,

$$
\left(-\mathrm{i} \gamma^{0} \partial_{t}-\mathrm{i} \gamma^{1} \partial_{x_{1}}-\mathrm{i} \gamma^{2} \partial_{x_{2}}-\mathrm{i} \gamma^{3} \partial_{x_{3}}+m_{0}\right) \psi=0,
$$


commonly abbreviated as $\left(-\mathrm{i} \not \partial+m_{0}\right) \psi=0$, where $\gamma^{0}, \gamma^{1}, \gamma^{2}, \gamma^{3}$, the Dirac matrices, are the $4 \times 4$ matrices given by

$$
\begin{aligned}
\gamma^{0} & =\left(\begin{array}{cccc}
1 & 0 & 0 & 0 \\
0 & 1 & 0 & 0 \\
0 & 0 & -1 & 0 \\
0 & 0 & 0 & -1
\end{array}\right), \gamma^{1}=\left(\begin{array}{cccc}
0 & 0 & 0 & 1 \\
0 & 0 & 1 & 0 \\
0 & -1 & 0 & 0 \\
-1 & 0 & 0 & 0
\end{array}\right), \\
\gamma^{2} & =\left(\begin{array}{cccc}
0 & 0 & 0 & -\mathrm{i} \\
0 & 0 & \mathrm{i} & 0 \\
0 & \mathrm{i} & 0 & 0 \\
-\mathrm{i} & 0 & 0 & 0
\end{array}\right), \gamma^{3}=\left(\begin{array}{cccc}
0 & 0 & 1 & 0 \\
0 & 0 & 0 & -1 \\
-1 & 0 & 0 & 0 \\
0 & 1 & 0 & 0
\end{array}\right) .
\end{aligned}
$$

The algebraic properties of the matrices $\gamma^{0}, \gamma^{1}, \gamma^{2}, \gamma^{3}$ allow us to obtain the following factorization of the Klein-Gordon equation:

$$
\left(\partial_{t}^{2}-\partial_{x_{1}}^{2}-\partial_{x_{2}}^{2}-\partial_{x_{3}}^{2}+m_{0}^{2}\right) \psi=\left(\mathrm{i} \not \partial+m_{0}\right)\left(-\mathrm{i} \not \partial+m_{0}\right) \psi=0
$$

Hence a solution to the Dirac equation is always a solution to the Klein-Gordon equation. The converse statement is not true.

\subsection{The Dirac equation in one spatial dimension}

As before, let $\Gamma$ be the hyperbola $x_{1} x_{2}=1$, and suppose $\mu \in \mathrm{AC}\left(\Gamma ; \Lambda_{\alpha, \beta}\right)$ for some positive reals $\alpha, \beta$. Then, in view of (1.2), the Fourier transform $\widehat{\mu}$ given by (1.1) solves the partial differential equation

$$
\left(\partial_{x_{1}} \partial_{x_{2}}+\pi^{2}\right) \widehat{\mu}=0
$$

in the sense of distribution theory. If we write $\psi(t, x):=\widehat{\mu}\left(\frac{1}{2}(t+x), \frac{1}{2}(t-x)\right)$, then $\psi$ solves the one-dimensional Klein-Gordon equation for a particle of mass $\pi$,

$$
\left(\partial_{t}^{2}-\partial_{x}^{2}+\pi^{2}\right) \psi=0 .
$$

Theorem 1.1 asserts that if $\alpha \beta>1$, there exists an infinite-dimensional space of solutions $\psi$ to (2.1) of the given form, subject to the condition of vanishing on

$$
\Lambda_{\alpha, \beta}^{\prime}=\left\{(m \alpha, m \alpha) \in \mathbb{R}^{2}: n \in \mathbb{Z}\right\} \cup\left\{(n \beta,-n \beta) \in \mathbb{R}^{2}: n \in \mathbb{Z}\right\} .
$$

The corresponding Dirac equation in this context is

$$
\left(-\mathrm{i} \sigma^{0} \partial_{t}-\mathrm{i} \sigma^{1} \partial_{x}+\pi\right) \psi=0
$$

where $\sigma^{0}, \sigma^{1}$ are the $2 \times 2$ matrices given by

$$
\sigma^{0}=\left(\begin{array}{cc}
1 & 0 \\
0 & -1
\end{array}\right) \quad \text { and } \quad \sigma^{1}=\left(\begin{array}{cc}
0 & 1 \\
-1 & 0
\end{array}\right)
$$

Here, $\psi=\left(\psi_{1}, \psi_{2}\right)$ in column form, and (2.2) may be written out more explicitly as the system

$$
\left\{\begin{array}{l}
-\mathrm{i} \partial_{t} \psi_{1}-\mathrm{i} \partial_{x} \psi_{2}+\pi \psi_{1}=0, \\
\mathrm{i} \partial_{t} \psi_{2}+\mathrm{i} \partial_{x} \psi_{1}+\pi \psi_{2}=0 .
\end{array}\right.
$$

The question pops up whether the Dirac equation (2.2) has an infinite-dimensional space of solutions $\psi=\left(\psi_{1}, \psi_{2}\right)$ that vanish on $\Lambda_{\alpha, \beta}^{\prime}$ for $\alpha \beta>1$. As both $\psi_{1}, \psi_{2}$ automatically solve the Klein-Gordon equation (this is a consequence of the factorization we mentioned previously in the context of three spatial dimensions), the natural requirement is that both $\psi_{1}, \psi_{2}$ are Fourier transform of measures in $\mathrm{AC}\left(\Gamma^{\prime}, \Lambda_{\alpha, \beta}^{\prime}\right)$. Here, $\Gamma^{\prime}$ is the hyperbola $t^{2}=x^{2}+1$, which corresponds to $\Gamma$ after the change of variables. From the assumptions made on $\psi_{1}, \psi_{2}$, we have that

$$
\psi_{j}(t, x)=\int_{-\infty}^{+\infty} f_{j}(v) \mathrm{e}^{\mathrm{i} \frac{1}{2} \pi\left[v(t+x)+v^{-1}(t-x)\right]} \mathrm{d} v, \quad j=1,2,
$$


where $f_{1}, f_{2}$ belong to $\mathcal{M}_{\beta}^{\perp}$ (this subspace of $L^{1}(\mathbb{R})$ is defined by (1.3)). Note that in the last step, we tacitly imposed the normalizing assumption that $\alpha=1$. As we implement this representation of $\psi_{1}, \psi_{2}$ into (2.3), we find that

$$
\int_{-\infty}^{+\infty}\left\{\left(v+v^{-1}+2\right) f_{1}(v)+\left(v-v^{-1}\right) f_{2}(v)\right\} \mathrm{e}^{\mathrm{i} \frac{1}{2} \pi\left[v(t+x)+v^{-1}(t-x)\right]} \mathrm{d} v=0
$$

and

$$
\int_{-\infty}^{+\infty}\left\{\left(v+v^{-1}-2\right) f_{2}(v)+\left(v-v^{-1}\right) f_{1}(v)\right\} \mathrm{e}^{\mathrm{i} \frac{1}{2} \pi\left[v(t+x)+v^{-1}(t-x)\right]} \mathrm{d} v=0 .
$$

As we plug in $t=x$, we see from the uniqueness theorem for the Fourier transform that the above two equations are equivalent to having

$$
\left(v+v^{-1}+2\right) f_{1}(v)+\left(v-v^{-1}\right) f_{2}(v)=0, \quad v \in \mathbb{R},
$$

and

$$
\left(v-v^{-1}\right) f_{1}(v)+\left(v+v^{-1}-2\right) f_{2}(v)=0, \quad v \in \mathbb{R},
$$

in the almost-everywhere sense. These requirements are compatible, as each one corresponds to having

$$
f_{2}(v)=\frac{1+v}{1-v} f_{1}(v), \quad v \in \mathbb{R}
$$

This means that we have reduced the study of the dimension of the space of solutions to the Dirac equation (2.2) subject to vanishing on $\Lambda_{\alpha, \beta}^{\prime}$ (with $\alpha=1$ ) plus the condition in terms of the Fourier transform to simply analyzing the dimension of the space

$$
\left\{f \in \mathcal{M}_{\beta}^{\perp}:(1+x)(1-x)^{-1} f(x) \text { is in } \mathcal{M}_{\beta}^{\perp}\right\} .
$$

To answer this dimension question we would need to better understand the structure of the preannihilator space $\mathcal{M}_{\beta}^{\perp}$.

\section{Perron-Frobenius operators}

\subsection{Dynamical systems}

The theory of dynamical systems deals with the time evolution of a system of points under a fixed change rule. An important feature of a dynamical system is its attractors, sets of points towards which the points of the system converge. A dynamical system is a four-tuple $(I, \mathfrak{S}, \mu, \tau)$, where $(I, \mathfrak{S}, \mu)$ is a measure space and $\tau: I \longrightarrow I$ is a measurable transformation. The measure $\mu$ is always positive and $\sigma$-finite; if it has finite total mass we renormalize and assume that the mass is 1 , so that $\mu$ becomes a probability measure. We denote by $\tau^{0}$ the identity map and write $\tau^{n}=\tau^{n-1} \circ \tau$ for $n=1,2,3, \ldots$. The evolution of a point $x \in I$ is described by its orbit under $\tau$, i.e., the sequence

$$
\left\{\tau^{n}(x)\right\}_{n=0}^{+\infty}
$$

In a concrete situation, the actual expression for the iterates $\tau^{n}$ tends to explode already for rather modest values of $n$, which makes it extremely difficult to extract substantial information based a direct approach. The most convenient approach is then the measure-theoretic one based on PerronFrobenius operators. If we have a random variable $X: I \longrightarrow \mathbb{R}$ distributed according to a density $\rho$, then the random variable $X \circ \tau$ will be distributed according to a new density, which is denoted by $\mathbf{P}_{\tau} \rho$. Instead of the orbits $\tau^{n}(x)$, we focus on the sequence of density functions

$$
\left\{\mathbf{P}_{\tau}^{n} \rho\right\}_{n=0}^{+\infty}
$$

The key point here is that, while $\tau$ may be nonlinear and discontinuous, the operator $\mathbf{P}_{\tau}$ is linear and bounded on the space $L^{1}(I, \mathfrak{S}, \mu)$ of integrable functions on $I$. The operator $\mathbf{P}_{\tau}$ is known as the Perron-Frobenius operator associated to the transformation $\tau$. It turns out that in most situations the sequence of density functions $\mathbf{P}_{\tau}^{n} \rho$ converges to certain densities of measures on $I$ that provide valuable information about the attractors of the system, which are known as invariant measures. More precisely, a $\sigma$-finite Borel measure $\nu$ on $I$ is said to be invariant under 
$\tau$ if $\nu\left(\tau^{-1}(A)\right)=\nu(A)$, for every $A \in \mathfrak{S}$. The densities of the $\mu$-absolutely continuous invariant measures can be recovered as eigenfunctions of the Perron-Frobenius operator corresponding to the eigenvalue $\lambda=1$. Perron-Frobenius operators appear in many branches of pure and applied mathematics, such as in stochastic processes, statistical mechanics, resonances, ordinary differential equations, thermodynamics, diffusion problems, positive matrices, and algorithms associated with continued fractions expansions. For a backgound on Perron-Frobenius operators, we refer to, e.g., see [3, 5], 9. In this work, we shall see how Perron-Frobenius operators are intimately related to the Heisenberg uniqueness pairs associated with the hyperbola $x_{1} x_{2}=1$. This leads to new and interesting questions concerning this important class of operators.

\subsection{Perron-Frobenius operators on bounded intervals}

In our situation, the dynamical systems involved are of the form $\left(I, \mathfrak{B}_{I}, m, \tau\right)$, where $I$ is a closed bounded interval of the real line, $m$ is the Lebesgue measure defined on $\mathfrak{B}_{I}$, the Borel $\sigma$-algebra of $I$, and $\tau$ denotes a measurable map from $I$ into itself. For $1 \leq p<+\infty$, the Banach space $L^{p}(I)$ consists of those measurable complex-valued (equivalence classes of) functions $f$ defined on $I$ for which the norm

$$
\|f\|_{L^{p}(I)}^{p}=\int_{I}|f|^{p} \mathrm{~d} m
$$

is finite. The space $L^{\infty}(I)$ consists of the essentially bounded measurable complex-valued functions $f$ supplied with the essential supremum norm. We shall use the following standard bilinear dual action:

$$
\langle f, g\rangle_{E}:=\int_{E} f g \mathrm{~d} m
$$

provided $f, g$ are Borel measurable, and $f g \in L^{1}(E)$. Here, $E \subset \mathbb{R}$ is a Borel set with positive linear measure: $m(E)>0$. For instance, if $f \in L^{1}(E)$ and $g \in L^{\infty}(E)$, the dual action is well-defined. When needed, we shall think of functions in $f \in L^{p}(E)$ as extended to all of $\mathbb{R}$ by setting them equal to 0 off $E$.

We shall need the following concepts.

Definition 3.1. The map $\tau: I \rightarrow I$ is said to be a filling $C^{2}$-smooth piecewise monotonic transformation if there exists a countable collection of pairwise disjoint open intervals $\left\{I_{u}\right\}_{u \in \mathcal{U}}$, such that

(i) the set $I \backslash \cup\left\{I_{u}: u \in \mathcal{U}\right\}$ has linear Lebesgue measure 0 ,

(ii) for any $u \in \mathcal{U}$, the restriction of $\tau$ to $I_{u}$ is strictly monotonic and extends to a $C^{2}$-smooth function on the closure of $I_{u}$, denoted $\tau_{u}$, and $\tau_{u}^{\prime} \neq 0$ holds in the interior of $I_{u}$,

(iii) for every $u \in \mathcal{U}, \tau_{u}$ maps the closure of $I_{u}$ onto $I$.

Definition 3.2. If, in the setting of Definition 3.1, all conditions are fulfilled, save that (iii) is replaced by the weaker condition $\left(i i^{\prime}\right)$ below, we say that $\tau$ is a partially filling $C^{2}$-smooth piecewise monotonic transformation:

$\left(i i i^{\prime}\right)$ there exists a $\delta>0$, such that for every $u \in \mathcal{U}$, the length of the interval $\tau\left(I_{u}\right)$ is $\geq \delta$.

In the context of the above two definitions, each intervals $I_{u}$ is known as a fundamental interval, and $\tau_{u}$ is said to be a branch. It is an important observation that each iterate $\tau^{n}$, with $n=1,2,3, \ldots$, has the same basic structure as the transformation $\tau$ itself. The fundamental intervals associated with $\tau^{n}$ are given by

$$
I_{\left(u_{1}, \ldots, u_{n}\right)}^{n}=\left\{x \in I: x \in I_{u_{1}}, \tau(x) \in I_{u_{2}}, \ldots, \tau^{n-1}(x) \in I_{u_{n}}\right\}, \quad\left(u_{1}, \ldots, u_{n}\right) \in \mathcal{U}_{\sharp}^{n},
$$

where $\mathcal{U}_{\sharp}^{n}$ consists of those elements $\left(u_{1}, \ldots, u_{n}\right) \in \mathcal{U}^{n}$ such that the above interval $I_{\left(u_{1}, \ldots, u_{n}\right)}^{n}$ becomes non-empty. The corresponding branch on $I_{\left(u_{1}, \ldots, u_{n}\right)}^{n}$ is denoted $\tau_{\left(u_{1}, \ldots, u_{n}\right)}^{n}=\tau_{u_{n}} \circ \cdots \circ \tau_{u_{1}}$.

The Koopman operator associated with $\tau$ is the composition operator which acts on $L^{\infty}(I)$ by the formula $\mathbf{C}_{\tau} g=g \circ \tau$. Clearly, $\mathbf{C}_{\tau}$ is linear and norm-contractive on $L^{\infty}(I)$. bounded. The 
Perron-Frobenius operator $\mathbf{P}_{\tau}: L^{1}(I) \rightarrow L^{1}(I)$ associated with $\tau$ is just the pre-adjoint of $\mathbf{C}_{\tau}$. Therefore, $\mathbf{P}_{\tau}$ is a norm contraction on $L^{1}(I)$ with

$$
\left\langle\mathbf{P}_{\tau} f, g\right\rangle_{I}=\left\langle f, \mathbf{C}_{\tau} g\right\rangle_{I}, \quad f \in L^{1}(I), g \in L^{\infty}(I) .
$$

It is immediate from (3.2) that an absolutely continuous measure $\mathrm{d} \mu_{f}=f \mathrm{~d} m$ is has the invariance property

if and only if

$$
\mu_{f}\left(\tau^{-1}(A)\right)=\mu(A) \text { for all } A \in \mathfrak{B}_{I}
$$

$$
\mathbf{P}_{\tau} f=f .
$$

It is clear that $\mathbf{C}_{\tau}^{n}=\mathbf{C}_{\tau^{n}}$, so, by duality we have that

$$
\mathbf{P}_{\tau}^{n}=\mathbf{P}_{\tau^{n}}, \quad n=1,2,3, \ldots
$$

Using (3.4), we find that

$$
\left(\mathbf{P}_{\tau} f\right)(x)=\sum_{u \in \mathcal{U}} J_{u}(x) f\left(\tau_{u}^{-1}(x)\right), \quad n=1,2,3, \ldots,
$$

where $J_{u} \geq 0$ is the function on $I$ that equals $\left|\left(\tau_{u}^{-1}\right)^{\prime}\right|$ on $\tau\left(I_{u}\right)$ and vanishes elsewhere. The map $J_{u}$ is well defined, since $\tau$ is piecewise strictly monotonic. By (3.5) we see that $\mathbf{P}_{\tau} f \geq 0$ for $f \geq 0$ and $\|\mathbf{P} f\|_{1}=\|f\|_{1}$. As $\mathbf{P}_{\tau}$ acts contractively on $L^{1}(I)$, its spectrum $\sigma\left(\mathbf{P}_{\tau}\right)$ is contained in the closed unit disk $\overline{\mathbb{D}}$.

\section{Perron-Frobenius operators for Gauss-type maps and invariant measures}

\subsection{The Gauss-type maps and the corresponding Perron-Frobenius operators}

For $t \in \mathbb{R}$, let $\{t\}_{1}$ be the number in the interval $\left[0,1\right.$ [ such that $t-\{t\}_{1} \in \mathbb{Z}$. We also need the expression $\{t\}_{2}$, which is in the interval $\left.]-1,1\right]$ and is uniquely determined by the requirement $t-\{t\}_{2} \in 2 \mathbb{Z}$. Next, for $0<\gamma<+\infty$, we consider the Perron-Frobenius operator for the transformation $\theta_{\gamma}(x):=\{\gamma / x\}_{1}$ on the unit interval $\left[0,1\left[\right.\right.$ (we put $\theta_{\gamma}(0):=0$ to define the map at the origin), which is given by

$$
\mathbf{P}_{\theta_{\gamma}} f(x)=\sum_{j=1}^{+\infty} \frac{\gamma}{(j+x)^{2}} f\left(\frac{\gamma}{j+x}\right), \quad x \in[0,1[,
$$

for $f \in L^{1}([0,1[)$, with the understanding that $f$ vanishes off $[0,1[$. It is easy to see that the eigenfunction equation

$$
\mathbf{P}_{\theta_{\gamma}} f=\lambda f, \quad|\lambda|=1,
$$

fails to have a solution $f$ in $L^{1}\left(\left[0,1[)\right.\right.$ for $0<\gamma<1$. In the case $\gamma=1, \mathbf{P}_{\theta_{1}}$ is the famous GaussKuzmin-Wirsing operator, which is connected with the continued fraction algorithm. It is known that $\mathbf{P}_{\theta_{1}} f=\lambda f$ with $|\lambda|=1$ has a non-trivial solution only for $\lambda=1$, in which case the solution $f$ is unique (up to a scalar multiple). These observations are basic in the proof of Theorem $\mathrm{B}$ (or, which is the same, Theorem $\left(\mathrm{B}^{\prime}\right)$; the natural parameter choices are $\alpha=2$ and $\beta=2 \gamma$.

For $0<\beta<+\infty$, we may instead consider the Perron-Frobenius operator for the transformation $\tau_{\beta}(x):=\{-\beta / x\}_{2}$ on the interval $\left.]-1,1\right]$ (we put $\tau_{\beta}(0):=0$ to define the map at the origin), which is given by

$$
\left.\left.\mathbf{P}_{\tau_{\beta}} f(x)=\sum_{j \in \mathbb{Z}^{\times}} \frac{\beta}{(2 j-x)^{2}} f\left(\frac{\beta}{2 j-x}\right), \quad x \in\right]-1,1\right],
$$

for $\left.\left.f \in L^{1}(]-1,1\right]\right)$, with the understanding that $f$ vanishes off $\left.]-1,1\right]$. Here, we use the standard notation $\mathbb{Z}^{\times}:=\mathbb{Z} \backslash\{0\}$. A rather elementary argument shows that the eigenfunction equation

$$
\mathbf{P}_{\tau_{\beta}} f=\lambda f, \quad|\lambda|=1,
$$


fails to have a solution $f$ in $\left.\left.L^{1}(]-1,1\right]\right)$ for $0<\beta<1$. For $\beta=1$, the transformation $\tau_{1}(x)=\{-1 / x\}_{2}$ is related to the continued fraction algorithm with even partial quotients, cf. [17, [26]. The map $\tau_{1}(x)=\{-1 / x\}_{2}$ has an indifferent fixed point at 1 . This entails that the invariant absolutely continuous density has infinite total mass; in this case, the density is given explicitly by $\left(1-x^{2}\right)^{-1}$. Using some ergodicity properties, it is easy to show that the equation $\mathbf{P}_{\tau_{1}} f=\lambda f$ fails to have solutions $f$ in $\left.\left.L^{1}(]-1,1\right]\right)$ for all $\lambda \in \mathbb{C}$ with $|\lambda|=1$. These observations are basic in the proof of Theorem $\mathrm{A}$ ( or, which is the same, Theorem $\mathrm{A}^{\prime}$ ).

\subsection{Discrete and singular invariant measures for the Gauss map}

It is well-known that the Gauss map $\theta_{1}(x):=\{1 / x\}_{1}$ on the unit interval $\left[0,1\right.$ [ [with $\theta_{1}(0):=0$ ] has infinitely many essentially different invariant measures. However, up to a constant multiple, there is only one that is absolutely continuous: $(1+x)^{-1} \mathrm{~d} x$. As for the discrete bounded invariant measures for $\theta_{1}$ there is an easy description. We write $\delta_{a}$ for the Dirac measure at point $a$. We shall need the set of fixed points of iterates of the Gauss map $\theta_{1}$ :

$$
\Sigma_{k}:=\left\{a \in \left[0,1\left[: \theta_{1}^{k}(a)=a\right\}, \quad \Sigma_{\infty}:=\bigcup_{k=1}^{+\infty} \Sigma_{k}\right.\right.
$$

For $a \in \Sigma_{\infty}$, there exists a minimal $k \geq 1$ such that $\theta_{1}^{k}(a)=a$; we write $k(a)$ for this $k$. We put

$$
\rho_{a}:=\frac{1}{k(a)} \sum_{j=0}^{k(a)-1} \delta_{\theta_{1}^{j}(a)}, \quad a \in \Sigma_{\infty} .
$$

Theorem 4.1. Let $\mu$ be a discrete bounded invariant measure for the Gauss map $\theta_{1}$. Then there is a function $\xi: \Sigma_{\infty} \rightarrow \mathbb{C}$ with $\sum_{a \in \Sigma_{\infty}}|\xi(a)|<+\infty$, such that

$$
\mu=\sum_{a \in \Sigma_{\infty}} \xi(a) \rho_{a}
$$

Proof. That the measure $\mu$ is invariant means that

$$
\int_{[0,1[} f \circ \theta_{1}(x) \mathrm{d} \mu(x)=\int_{[0,1[} f(x) \mathrm{d} \mu(x)
$$

for every $f$ integrable with respect to $|\mu|$. It is trivial to check that all measures of the given form are invariant. In the other direction, it is well-known that every discrete invariant measure $\mu$ may be decomposed into irreducible (ergodic) parts. We just need to show that up to a multiplicative constant, each irreducible part is of the form $\rho_{a}$. So, let $\rho$ be an ergodic discrete invariant probability measure on $[0,1$ [. Let $E \subset[0,1$ [ be the minimal countable set which carries the mass of $\rho$. By the Birkhoff-Khinchin Ergodic Theorem, we have for each $x_{0} \in E$ that

$$
\frac{1}{n} \sum_{j=0}^{n-1} f\left(\theta_{1}^{j}\left(x_{0}\right)\right) \rightarrow \int_{E} f \mathrm{~d} \rho \quad \text { as } n \rightarrow+\infty .
$$

In particular, if we let $f$ equal the characteristic function of the one-point set $\left\{x_{0}\right\}$, and observe that from the minimality of $E$, we have $\rho\left(\left\{x_{0}\right\}\right)>0$, we find that approximately a $\rho\left(\left\{x_{0}\right\}\right)$ proportion of the time on the interval $0 \leq j \leq n-1$, we have $\theta_{\gamma}^{j}\left(x_{0}\right)=x_{0}$. This means that $x_{0} \in \Sigma_{\infty}$. We may also conclude by picking other functions $f$ that $E$ equals the orbit of $x_{0}$ :

$$
E=\left\{\theta_{\gamma}^{j}\left(x_{0}\right): 0 \leq j \leq k\left(x_{0}\right)-1\right\}
$$

By ergodicity, each point of $E$ must have equal mass, so that $\rho=\rho_{a}$ with $a=x_{0}$. This completes the proof.

Remark 4.2. (a) The proof of Theorem 4.1 did not really use the fact that we are dealing with the Gauss map. In particular, the corresponding assertion holds for the transformation $\tau_{1}(x)=$ $\{-1 / x\}_{2}$ in place of the Gauss map.

(b) The set $\Sigma_{\infty}$ consists of the quadratic surds plus the origin. 
(c) The discrete measures provided by Theorem 4.1 above extend in a natural fashion to the positive half-axis $\mathbb{R}_{+}$. These extensions lift to the hyperbola branch $\Gamma_{+}$where $x_{1} x_{2}=1$ and $x_{1}>0$, and the lifted measures on $\Gamma_{+}$have Fourier transforms that vanish on the lattice-cross $\Lambda_{\alpha, \beta}$ with $\alpha=\beta=2$. Indeed, this is the only way to obtain such discrete measures on $\Gamma_{+}$.

\subsection{The Minkowski measure}

The most studied singular continuous measure for the Gauss-Kuzmin-Wirsing operator is the Minkowski measure. It belongs to a family of singular probability measures which we call Markovian measures (see below). The Minkowski question mark function was first introduced by Minkowski in 1904. Let $\left\{a_{n}(x)\right\}_{n=1}^{+\infty}$ be the sequence of positive integers in the continued fraction expansion of $x$. Salem 23. proved that the question mark function can be defined by

$$
?(x)=\sum_{j=1}^{+\infty} \frac{2(-1)^{j+1}}{2^{a_{1}(x)+a_{2}(x)+\cdots+a_{j}(x)}}, \quad 0 \leq x \leq 1,
$$

where the series is finite for rational $x$. Then ? is a strictly increasing continuous singular function. It takes the rational numbers into the dyadic numbers and the quadratic surds into the rationals. The Riemann-Stieltjes measure $\mathrm{d}$ ? on $[0,1]$, is then a singular continuous probability measure, which can be shown to be invariant for the Gauss map $\theta_{1}(x)=\{1 / x\}_{1}$. The numbers $a_{j}(x) \in \mathbb{Z}_{+}$ are the successive remainders which we throw away as we iterate the Gauss map at a point $x$. Let us say that a probability measure $\mu$ on $[0,1]$ is Markovian with respect to the Gauss map if there are numbers $q(j)$ with $0 \leq q(j)<1$ and

$$
\sum_{j=1}^{+\infty} q(j)=1,
$$

such that the $\mu$-mass of the "cylinder set"

$$
\left\{x \in \left[0,1\left[: a_{j}(x)=b_{j} \text { for } j=1, \ldots, k\right\}\right.\right.
$$

equals

$$
\prod_{j=1}^{k} q\left(b_{j}\right)
$$

Then the Minkowski measure is Markovian with $q(j)=2^{-j}$. Problem (d) in [12] could be settled in the negative if we could find a singular continuous measure on $\Gamma$, the Fourier transform of which tends to zero at infinity, while it vanishes along $\Lambda_{\alpha, \beta}$ with $\alpha=\beta=1$. A perhaps easier task is to find a singular invariant measure on $[0,1]$ with respect to the Gauss map such that the Fourier transform tends to zero at infinity (measures whose Fourier transforms decay to 0 at infinity are called Rajchman measures). The Markovian measures are all invariant and singular continuous. E.g., the Minkowski measure is of this type. But it is not known if it is Rajchman. Indeed, this question is a well-known problem raised by Salem 23 .

\section{Further properties of Perron-Frobenius operators}

\subsection{The spectral decomposition of Perron-Frobenius operators}

Let $I$ be a closed bounded interval. The total variation of a complex-valued function $h: I \longrightarrow \mathbb{C}$ is

$$
\operatorname{var}_{I}(h)=\sup \left\{\sum_{j=1}^{n-1}\left|h\left(t_{j+1}\right)-h\left(t_{j}\right)\right|\right\},
$$

where the supremum is taken over all $t_{1}, \ldots, t_{n} \in I$ with $t_{1}<\ldots<t_{n}$. The function $h$ is said to be of bounded variation when $\operatorname{var}_{I}(h)<+\infty$. We will denote by $\operatorname{BV}(I)$ the subspace of $L^{1}(I)$ 
functions, which has a representative of bounded variation. The space $\mathrm{BV}(I)$ becomes a Banach space when supplied, e.g., with the norm

$$
\|h\|_{\mathrm{BV}}=\|h\|_{1}+\inf _{\tilde{h} \sim h} \operatorname{var}_{I}(\tilde{h}), \quad h \in \mathrm{BV}(I),
$$

where the infimum is taken over the elements in the equivalence class of $h$ (so that $\tilde{h}=h$ except on a Lebesgue null set). It is well-known that for each $h \in \mathrm{BV}(I)$ there is a right-continuous function in the class of $h$ where the infimum in the definition of $\|h\|_{\mathrm{BV}}$ in (5.1) is attained. In particular, $B V(I)$ is a subspace of $L^{\infty}(I)$, see [16. A perhaps more precise description of $\mathrm{BV}(I)$ is that these are the primitive functions of the finite complex-valued Borel measures on $I$.

Let $\mathbb{T}=\{z \in \mathbb{C}:|z|=1\}$ denote the unit circle in $\mathbb{C}$ and let $\sigma_{\mathrm{p}}\left(\mathbf{P}_{\tau}\right)$ denote the point spectrum of $\mathbf{P}_{\tau}$, where the Perron-Frobenius operator $\mathbf{P}_{\tau}$ is thought of as acting on $L^{1}(I)$. As a consequence of the Ionescu-Tulcea and Marinescu theorem, see [5] and [16], Section 5.3, the following spectral decomposition holds for $\mathbf{P}_{\tau}$. We recall the notions of filling and partially filling $C^{2}$-smooth monotonic transformations in Definitions 3.1 and 3.2 .

Theorem C. Suppose $\tau: I \rightarrow I$ is a partially filling $C^{2}$-smooth piecewise monotonic transformation with the following properties $(i)-($ ii):

(i) [uniform expansiveness] There exist and integer $m \geq 1$ and a positive real $\epsilon$ such that $\left|\left(\tau^{m}\right)^{\prime}(x)\right| \geq 1+\epsilon$ for all $x \in \cup\left\{I_{u}: u \in \mathcal{U}_{\sharp}^{m}\right\}$.

(ii) [second derivative condition] There exists a positive constant $M$ such that $\left|\tau^{\prime \prime}(x)\right| \leq M\left|\tau^{\prime}(x)\right|^{2}$ for all $x \in \cup\left\{I_{u}: u \in \mathcal{U}\right\}$.

Then $\Lambda_{\tau}:=\sigma_{\mathrm{p}}\left(\mathbf{P}_{\tau}\right) \cap \mathbb{T}$ is finite and non-empty, say $\Lambda_{\tau}=\left\{\lambda_{1}, \ldots, \lambda_{p}\right\}$. Here, one of the eigenvalues is the point 1 , say $\lambda_{1}=1$. If $E_{i}$ denotes the eigenspace of $\mathbf{P}_{\tau}$ corresponding to $\lambda_{i}$, then $E_{i}$ is finitedimensional and $E_{i} \subset \mathrm{BV}(I)$. In addition, we have

$$
\mathbf{P}_{\tau}^{n} h=\sum_{i=1}^{p} \lambda_{i}^{n} \mathbf{P}_{\tau, i} h+\mathbf{Z}_{\tau}^{n} h, \quad h \in L^{1}(I), \quad n=1,2,3, \ldots,
$$

where the operators $\mathbf{P}_{\tau, i}$ are the projections onto $E_{i}$, and the operator $\mathbf{Z}_{\tau}$ acts boundedly on on $L^{1}(I)$ as well as on $\mathrm{BV}(I)$. Moreover, the spectrum of $\mathbf{Z}_{\tau}$ as an operator acting on $\mathrm{BV}(I)$ is contained in the open unit disk $\mathbb{D}$; i.e., $\mathbf{Z}_{\tau}$ acting on $\mathrm{BV}(I)$ has spectral radius $<1$.

Remark 5.1. (a) It is a by-product of Theorem $\mathrm{C}$ that $\mathbf{P}_{\tau}$ acts boundedly on BV(I). In fact, the way things work is that this rather elementary observation is the beginning of the analysis that leads up to Theorem C,

(b) Since 1 is an eigenvalue of $\mathbf{P}_{\tau}$, the corresponding eigenfunction (which is then in BV $(I)$ ) is the density for an invariant measure. If there are several such eigenfunction for $\lambda_{1}=1$, then one of them is $\geq 0$, which we can normalize so that we get an absolutely continuous invariant probability measure with density in $\mathrm{BV}(I)$; compare with the proof of Theorem 7.2 .

(c) By a theorem of Rota (see [5, 24]), the exterior eigenvalues $\left\{\lambda_{1}, \ldots, \lambda_{p}\right\}$ form a finite union of cyclic subgroups of $\mathbb{T}$. In particular, they are all roots of unity, that is, $\lambda_{i}^{N}=1$ holds for all $i=1, \ldots, p$, for some big enough positive integer $N$.

(d) The formulation of the Ionescu-Tulcea and Marinescu theorem in [16] initially assumes that $\tau$ is "filling", but it is later remarked that the theorem holds for "partially filling" transformations (cf. Definitions 3.1 and 3.2); see [16], p. 214, and also [6] and [8, p. 169.

(e) When considered as an operator on $L^{1}(I)$, the Perron-Frobenius operator $\mathbf{P}_{\tau}$ will usually have eigenvalues at all points of the open disk, with eigenfunctions in $L^{\infty}([-1,1])$ (cf. [18).

Remark 5.2. From the presentation in 16, Section 5.3, it is clear that if $\tau$ is "filling", we have a stronger assertion in Theorem $\mathbb{C}: \lambda_{1}=1$ is the only eigenvalue of $\mathbf{P}_{\tau}$ on $\mathbb{T}$, and that the $\tau$ invariant absolutely continuous probability measure is unique, with a density that is bounded from above and below by two positive constants. Cf. also [8, p. 172, where it is shown that under the given assumptions, $\tau$ is mixing. We briefly outline the argument, following the presentation in [16, Section 5.3. We write $f_{u}:=\tau_{u}^{-1}: I \rightarrow \bar{I}_{u}$ for the inverse branches $(u \in \mathcal{U})$. The assumptions $(i)$ 
and $(i i)$ of Theorem correspond to the conditions $\left(\mathrm{E}_{m}\right)$ and $(\mathrm{A})$ of [16], pp. 191-192. Next, by [16], Proposition 5.3.3, we have that $\left(\mathrm{E}_{m}\right)$ forces $\left|f_{u}^{\prime}(x)\right|$ to be uniformly bounded in $x \in I$ and $u \in \mathcal{U}$. In view of Condition (A) of [16], p. 192, we also know that $\left|f_{u}^{\prime \prime}(x)\right|$ is uniformly bounded in $x \in I$ and $u \in \mathcal{U}$. In particular, then, $f_{u}^{\prime}$ is absolutely continuous for all $u \in \mathcal{U}$. Next, by [16], Proposition 5.3.4, we use this absolute continuity together with condition $\left(\mathrm{E}_{m}\right)$ of [16], p. 191, to see that condition (C) [Rényi's distortion estimate] holds as well. Next, by [16], Theorem 4.3.5, we use condition $(\mathrm{C})$ to get that there is a unique ergodic $\tau$-invariant absolutely continuous probability measure, and that its density is bounded from above and below by positive constants. This means that there is only one eigenvalue, namely 1 . We mention here that the condition (BV) of [16, p. 200 - which requires the sum of the variation of $f_{u}^{\prime}$ over $u \in \mathcal{U}$ to be bounded - is a trivial consequence of condition (A), as the sum of the variances of $f_{u}^{\prime}$ over $u \in \mathcal{U}$ amounts to summing the lengths of the intervals $I_{u}$, which all add up to the length of $I$.

\subsection{The Folklore theorem (Adler's theorem)}

We shall be interested in partially filling $C^{2}$-smooth monotonic transformations of a closed bounded interval $I$. In view of Remark $5.1(d)$, we can be assured that Theorem C] holds also in this more general situation. Moreover, Remark 5.1 (b) tells us that there exists a $\tau$-invariant absolutely continuous probability measure, but it might not be unique. To get uniqueness, we need to make stronger conditions on $\tau$. In this direction we have Adler's Theorem, also known as the Folklore theorem (see [5]).

Theorem D (Adler's theorem). Let $\tau$ be a partially filling $C^{2}$-smooth piecewise monotonic transformation with the following properties $(i)-(i v)$ :

(i) [uniform expansiveness] There exist an integer $m \geq 1$ and a positive real $\epsilon$ such that $\left|\left(\tau^{m}\right)^{\prime}(x)\right| \geq$ $1+\epsilon$ for all $x \in \cup\left\{I_{u}: u \in \mathcal{U}_{\sharp}^{n}\right\}$.

(ii) [second derivative condition] There exists a positive constant $M$ such that $\left|\tau^{\prime \prime}(x)\right| \leq M\left|\tau^{\prime}(x)\right|^{2}$ for all $x \in \cup\left\{I_{u}: u \in \mathcal{U}\right\}$.

(iii) [Markov property 1] For every $u \in \mathcal{U}$ there is $n=n(u) \geq 1$ such that $\operatorname{clos}\left[\tau^{n}\left(I_{u}\right)\right]=I$.

(iv) [Markov property 2] Whenever $\tau\left(I_{u}\right) \cap I_{v} \neq \emptyset$ holds for two indices $u, v \in \mathcal{U}$, then $\tau\left(I_{u}\right) \supset I_{v}$.

Then $\tau$ admits a unique absolutely continuous invariant probability measure $\mathrm{d} \rho=\varrho \mathrm{d} m$. Moreover, the density $\varrho$ is bounded from above and below by positive constants.

Remark 5.3. (a) A transformation $\tau$ satisfying (iii)-(iv) above is said to be a Markov map. (b) A well-known result which preceded Adler's theorem is the Lasota and Yorke theorem [19].

\subsection{Dynamical properties of the Gauss-type maps}

We first consider the transformation $\tau_{\beta}$ of the interval $\left.]-1,1\right]$, as defined by $\tau_{\beta}(0):=0$ and by

$$
\tau_{\beta}(x):=\left\{-\frac{\beta}{x}\right\}_{2}, \quad x \neq 0 .
$$

Here, we recall that for $t \in \mathbb{R},\{t\}_{2}$ denotes the uniquely determined number in ]-1,1] with with $t-\{t\}_{2} \in 2 \mathbb{Z}$. We restrict our attention to $\beta>1$ only. Let the index set $\mathcal{U}=\mathcal{U}_{\beta}$ be the subset of the non-zero integers $u$ for which the corresponding branch interval is non-empty:

$$
\left.I_{u}:=\right] \frac{\beta}{2 u+1}, \frac{\beta}{2 u-1}[\cap]-1,1[\neq \emptyset \text {. }
$$

We put $u_{0}=u_{0}(\beta):=\frac{1}{2}\left(\beta-\{\beta\}_{2}\right)$, which is an integer $\geq 1$. We note that if $\beta$ is an odd integer, then

$$
\left.I_{u}=\right] \frac{\beta}{2 u+1}, \frac{\beta}{2 u-1}[, \quad u \in \mathcal{U},
$$

and $\mathcal{U}$ consists of all $u \in \mathbb{Z}^{\times}$with $|u| \geq \frac{1}{2}(\beta+1)$. In this case, the "filling" requirement is fulfilled: $\left.\tau_{\beta}\left(I_{u}\right)=\right]-1,1[$ holds for all $u \in \mathcal{U}$. More generally, when $\beta$ is not an odd integer, then $\mathcal{U}$ consists 
of all $u \in \mathbb{Z}^{\times}$with $|u| \geq u_{0}$, and we have

$$
\left.I_{u}=\right] \frac{\beta}{2 u+1}, \frac{\beta}{2 u-1}\left[, \quad u \in \mathcal{U} \backslash\left\{ \pm u_{0}\right\} .\right.
$$

so that

$$
\left.\tau_{\beta}\left(I_{u}\right)=\right]-1,1\left[, \quad u \in \mathcal{U} \backslash\left\{ \pm u_{0}\right\} .\right.
$$

We see that the deviation from the "filling" requirement is rather slight (just two branches fail).

Next, we quickly calculate the derivative of $\tau_{\beta}$ :

$$
\tau_{\beta}^{\prime}(x)=\frac{\beta}{x^{2}} \geq \beta>1, \quad x \in \cup\left\{I_{u}: u \in \mathcal{U}\right\},
$$

so the uniform expansiveness condition is met already by $\tau_{\beta}$ (with $m=1$ ). Moreover,

$$
\frac{\left|\tau_{\beta}^{\prime \prime}(x)\right|}{\left|\tau_{\beta}^{\prime}(x)\right|^{2}} \leq \frac{2|x|}{\beta} \leq 2, \quad x \in \cup\left\{I_{u}: u \in \mathcal{U}\right\},
$$

so we also have the second derivative control. Unfortunately, we cannot rely on Theorem $\mathrm{C}$ to give us the uniqueness of the absolutely continuous invariant measure for $\tau_{\beta}$, as it apparently allows for non-uniqueness (although Remark 5.2 says that in the "filling" case we really do have the needed uniqueness). We also cannot rely on Adler's theorem, as $\tau_{\beta}$ is not necessarily a Markov map. We will check that condition (iii) of Adler's theorem holds for all sufficiently big $n$, say $n \geq n(u)$. and

We are also interested in the Gauss-type map $\theta_{\gamma}$ of the interval $\left[0,1\left[\right.\right.$, as defined by $\theta_{\gamma}(0):=0$

$$
\theta_{\gamma}(x):=\left\{\frac{\gamma}{x}\right\}_{1}, \quad x \neq 0 .
$$

Here, we recall that $\{t\}_{1}$ is the fractional part of $t \in \mathbb{R}$, with values in $\left[0,1\left[\right.\right.$ and $t-\{t\}_{1} \in \mathbb{Z}$. We restrict our attention to $\gamma>1$ only. Let the index set $\mathcal{V}=\mathcal{V}_{\gamma}$ be the subset of the positive integers $v$ for which the corresponding branch interval is non-empty:

$$
\left.J_{v}:=\right] \frac{\gamma}{v+1}, \frac{\gamma}{v}[\cap] 0,1[\neq \emptyset .
$$

We note that if $\gamma$ is an integer, then $\mathcal{V}$ consists of all positive integers $v$ with $v \geq \gamma$, and

$$
\left.J_{v}=\right] \frac{\gamma}{v+1}, \frac{\gamma}{v}[, \quad v \in \mathcal{V} .
$$

More generally, if $v_{0}=v_{0}(\gamma):=\gamma-\{\gamma\}_{1} \in \mathbb{Z}_{+}$, then

$$
\left.J_{v}=\right] \frac{\gamma}{v+1}, \frac{\gamma}{v}\left[, \quad v \in \mathcal{V} \backslash\left\{v_{0}\right\},\right.
$$

and

$$
\left.\theta_{\gamma}\left(J_{v}\right)=\right] 0,1\left[, \quad u \in \mathcal{V} \backslash\left\{v_{0}\right\}\right.
$$

So, the deviation from the "filling" requirement is rather slight (only one branch fails). Next, we quickly calculate the derivative of $\theta_{\gamma}$ :

$$
\left|\theta_{\beta}^{\prime}(x)\right|=\frac{\gamma}{x^{2}} \geq \beta>1, \quad x \in \cup\left\{J_{v}: v \in \mathcal{U}\right\}
$$

so the uniform expansiveness condition is met already by $\theta_{\beta}$ (with $m=1$ ). Moreover,

$$
\frac{\left|\theta_{\gamma}^{\prime \prime}(x)\right|}{\left|\theta_{\gamma}^{\prime}(x)\right|^{2}} \leq \frac{2 x}{\gamma} \leq 2, \quad x \in \cup\left\{J_{v}: v \in \mathcal{V}\right\},
$$

so we also have the second derivative control. Again, we cannot unfortunately rely on Theorem $\mathrm{C}$ to give us the uniqueness of the absolutely continuous invariant probability measure for $\theta_{\gamma}$, as it apparently allows for non-uniqueness (although Remark 5.2 says that in the "filling" case we have the needed uniqueness). We also cannot rely on Adler's theorem, as $\theta_{\gamma}$ is not necessarily a Markov map. However, it appears that here, it is nevertheless known that the absolutely continuous $\theta_{\gamma^{-}}$ invariant probability measure is unique and has strictly positive density almost everywhere. One 
way to do this is to check that condition (iii) of Adler's theorem is fulfilled for all $n \geq n(u)$, and proceed in an analogous fashion as we do for $\tau_{\beta}$, with $\beta>1$. We omit the details.

\subsection{The explicit calculation of invariant measures}

In general, the computation of the absolutely continuous invariant measures for $\tau_{\beta}$ (as well as for $\theta_{\gamma}$ ) is intractable. Only in a few particular cases is it possible to supply explicit expressions for the corresponding densities. They all correspond to values of the parameters for which we are dealing with Markov maps. For instance, when $\beta>1$ is an odd integer, $\tau_{\beta}$ is "filling" [i.e., we have complete branches], and the unique $\tau_{\beta}$-invariant probability measure on $[-1,1]$ is given by

$$
\frac{c(\beta)}{1-(x / \beta)^{2}} 1_{[-1,1]}(x) \mathrm{d} x, \quad \text { where } \quad \frac{1}{c(\beta)}=\int_{-1}^{1} \frac{\mathrm{d} x}{1-(x / \beta)^{2}}=\frac{\beta}{2} \log \frac{\beta+1}{\beta-1} \quad(\beta=3,5,7, \ldots) .
$$

It is more interesting that it is possible to obtain the $\tau_{\beta}$-invariant probability density in a more complicated situation, when $\beta=\frac{3}{2}$. The uniqueness and ergodicity of that measure will be obtained in Section 7. As for notation, we write $1_{E}$ for the characteristic function of a set $E \subset \mathbb{R}$, which equals 1 on $E$ and vanishes elsewhere.

Proposition 5.4. $(\beta=3 / 2)$ The density of the unique ergodic $\tau_{3 / 2}$-invariant absolutely continuous probability measure is given by

$$
\varrho_{0}(x)=c_{0}\left\{\frac{1}{1-(2 x / 3)^{2}} 1_{\left[-\frac{1}{2}, \frac{1}{2}\right]}(x)+\frac{3 / 4}{(1-|x| / 3)(1+2|x| / 3)} 1_{[-1,1] \backslash\left[-\frac{1}{2}, \frac{1}{2}\right]}(x)\right\},
$$

where $c_{0}^{-1}=\frac{3}{2} \log (5 / 2)$.

Proof. To simplify the notation, we write $\varrho_{1}(x):=c_{0}^{-1} \varrho_{0}(x)$, so that $\varrho_{1}(x)$ stands for the bracketed expression. We note that both $\varrho_{0}, \varrho_{1}$ are even functions. We need to check that

$$
\sum_{j \in \mathbb{Z}^{\times}} \frac{3 / 2}{(2 j-x)^{2}} \varrho_{1}\left(\frac{3 / 2}{2 j-x}\right)=\varrho_{1}(x), \quad x \in[-1,1],
$$

where this equality should be understood in the almost-everywhere sense. Since

$$
\frac{3 / 2}{2 j-x} \in\left[-\frac{1}{2}, \frac{1}{2}\right] \quad \text { for } \quad|j| \geq 2, \quad x \in[-1,1],
$$

we may evaluate the sum of all but two terms in the left-hand side of (5.8), as most of the terms cancel:

$$
\begin{aligned}
\sum_{j:|j| \geq 2} \frac{3 / 2}{(2 j-x)^{2}} \varrho_{1}\left(\frac{3 / 2}{2 j-x}\right)=\sum_{j:|j| \geq 2} \frac{3 / 2}{(2 j-x)^{2}} \times \frac{1}{1-\frac{1}{(2 j-x)^{2}}} & \\
=\sum_{j:|j| \geq 2} \frac{3 / 2}{(2 j-x)^{2}-1}= & \frac{3}{4} \sum_{j:|j| \geq 2}\left[\frac{1}{2 j-x-1}-\frac{1}{2 j-x+1}\right] \\
& =\frac{3}{4}\left[\frac{1}{3-x}+\frac{1}{3+x}\right]=\frac{1 / 2}{1-(x / 3)^{2}}, \quad x \in[-1,1] .
\end{aligned}
$$

Next, we see that

$$
\left.\left.\left.\left.\frac{3 / 2}{2-x} \in\right] \frac{1}{2}, 1\right], \quad x \in\right]-1, \frac{1}{2}\right]
$$


and that this expression is in $\left.] 1, \frac{3}{2}\right]$ for $\left.\left.x \in\right] \frac{1}{2}, 1\right]$. Here, we may of course replace $x$ by $-x$ if we make the necessary adjustments. It follows that

$$
\begin{gathered}
\sum_{j:|j|=1} \frac{3 / 2}{(2 j-x)^{2}} \varrho_{1}\left(\frac{3 / 2}{2 j-x}\right)=\frac{3 / 2}{(2-x)^{2}} \varrho_{1}\left(\frac{3 / 2}{2-x}\right)+\frac{3 / 2}{(2+x)^{2}} \varrho_{1}\left(-\frac{3 / 2}{2+x}\right) \\
=\frac{3 / 2}{(2-x)^{2}} \times \frac{3 / 4}{\left(1-\frac{1 / 2}{2-x}\right)\left(1+\frac{1}{2-x}\right)} 1_{\left[-1, \frac{1}{2}\right]}(x)+\frac{3 / 2}{(2+x)^{2}} \times \frac{3 / 4}{\left(1-\frac{1 / 2}{2+x}\right)\left(1+\frac{1}{2+x}\right)} 1_{\left[-\frac{1}{2}, 1\right]}(x) \\
\left.=\frac{1 / 4}{\left(1-\frac{2 x}{3}\right)\left(1-\frac{x}{3}\right)} 1_{\left[-1, \frac{1}{2}\right]}(x)+\frac{1 / 4}{\left(1+\frac{2 x}{3}\right)\left(1+\frac{x}{3}\right)} 1_{\left[-\frac{1}{2}, 1\right]}(x) \quad x \in\right]-1,1[,
\end{gathered}
$$

We may now express the whole sum in (5.8):

$$
\begin{gathered}
\sum_{j \in \mathbb{Z} \times} \frac{3 / 2}{(2 j-x)^{2}} \varrho_{1}\left(\frac{3 / 2}{2 j-x}\right)=\frac{1 / 2}{1-\left(\frac{x}{3}\right)^{2}}+\frac{1 / 4}{\left(1-\frac{2 x}{3}\right)\left(1-\frac{x}{3}\right)} 1_{\left[-1, \frac{1}{2}\right]}(x)+\frac{1 / 4}{\left(1+\frac{2 x}{3}\right)\left(1+\frac{x}{3}\right)} 1_{\left[-\frac{1}{2}, 1\right]}(x) \\
=\frac{1}{4}\left[\frac{1}{1-\frac{x}{3}}+\frac{1}{1+\frac{x}{3}}\right]+\frac{1}{4}\left[\frac{2}{1-\frac{2 x}{3}}-\frac{1}{1-\frac{x}{3}}\right] 1_{\left[-1, \frac{1}{2}\right]}(x)+\frac{1}{4}\left[\frac{2}{1+\frac{2 x}{3}}-\frac{1}{1+\frac{x}{3}}\right] 1_{\left[-\frac{1}{2}, 1\right]}(x) \\
\left.=\frac{1}{4}\left[\frac{2}{1-\frac{2 x}{3}}+\frac{2}{1+\frac{2 x}{3}}\right] 1_{\left[-\frac{1}{2}, \frac{1}{2}\right]}(x)+\frac{1}{4}\left[\frac{1}{1-\frac{x}{3}}+\frac{2}{1+\frac{2 x}{3}}\right] 1_{] \frac{1}{2}, 1\right]}(x)+\frac{1}{4}\left[\frac{1}{1+\frac{x}{3}}+\frac{2}{1-\frac{2 x}{3}}\right] 1_{]-1,-\frac{1}{2}[(x)}(x) \quad x \in\right]-1,1[. \\
=\frac{1 / 2}{1-\left(\frac{2 x}{3}\right)^{2}} 1_{\left[-\frac{1}{2}, \frac{1}{2}\right]}(x)+\frac{3 / 4}{\left(1-\frac{|x|}{3}\right)\left(1+\frac{2|x|}{3}\right)} 1_{[-1,1] \backslash\left[-\frac{1}{2}, \frac{1}{2}\right]}(x)=\varrho_{1}(x), \quad x
\end{gathered}
$$

The constant $c_{0}$ is determined by the requirement that we should have a probability density and easily computed. The proof is complete.

Remark 5.5. (a) It is possible to establish with similar means the $\tau_{\beta}$-invariant absolutely continuous probability measure for $\beta=n(2 n+1) /(n+1)$, where $n$ is a positive integer.

(b) We mention here that the analogous $\theta_{\gamma}$-invariant absolutely continuous probability measures are known explicitly for $\gamma \in \mathbb{Z}_{+}$, see, e.g., [7]:

$$
\frac{c(\gamma)}{1+x / \gamma} 1_{[0,1]}(x) \mathrm{d} x, \quad \text { where } \quad \frac{1}{c(\gamma)}=\int_{0}^{1} \frac{\mathrm{d} x}{1+x / \gamma}=\gamma \log (1+1 / \gamma) \quad(\gamma=1,2,3, \ldots) .
$$

\section{Characterization of the pre-annihilator space $\mathcal{M}_{\beta}^{\perp}$}

\subsection{Purpose of the section; some notation}

In this section we provide a characterization of the subspace $\mathcal{M}_{\beta}^{\perp}$ in terms of certain operators. We proceed in a fashion somewhat similar to that the one used in the proof of Lemma 5.2 in [12. We

recall from Subsection 3.2 that the Koopman operator for $\tau_{\beta}$ is denoted by $\mathbf{C}_{\tau_{\beta}}$, and recall from Subsection 4.1 that the corresponding Perron-Frobenius operator is $\mathbf{P}_{\tau_{\beta}}: L^{1}([-1,1]) \rightarrow L^{1}([-1,1])$, given by

$$
\mathbf{P}_{\tau_{\beta}} h(x)=\sum_{j \in \mathbb{Z}^{\times}} \frac{\beta}{(2 j-x)^{2}} h\left(\frac{\beta}{2 j-x}\right), \quad x \in[-1,1],
$$

with the understanding that $h \in L^{1}([-1,1])$ vanishes off the interval $[-1,1]$. Following the notation of [12], we denote by $L_{2}^{\infty}(\mathbb{R})$ the subspace of $L^{\infty}(\mathbb{R})$ of 2-periodic functions, which is the same as the weak-star closure of

$$
\operatorname{span}\left\{\mathrm{e}^{i n \pi x}: n \in \mathbb{Z}\right\}
$$

Likewise, we let $L_{\langle\beta\rangle}^{\infty}(\mathbb{R})$ be the weak-star closure of

$$
\operatorname{span}\left\{\mathrm{e}^{i n \pi \beta / x}: n \in \mathbb{Z}\right\},
$$


which also has a characterization in terms of periodicity $\left[f \in L_{\langle\beta\rangle}^{\infty}(\mathbb{R})\right.$ if and only if the function $f(\beta / x)$ is in $\left.L_{2}^{\infty}(\mathbb{R})\right]$. Let $\mathbf{S}: L^{\infty}([-1,1]) \longrightarrow L^{\infty}(\mathbb{R} \backslash[-1,1])$ be the operator defined by

$$
\mathbf{S} g(x)=g\left(\{x\}_{2}\right), \quad x \in \mathbb{R} \backslash[-1,1]
$$

and let $\mathbf{T}: L^{\infty}(\mathbb{R} \backslash[-\beta, \beta]) \longrightarrow L^{\infty}([-\beta, \beta])$ be the operator given by

$$
\mathbf{T} g(x)=g\left(-\frac{\beta}{\{-\beta / x\}_{2}}\right), \quad x \in[-\beta, \beta] \backslash\{0\} .
$$

It is clear that $\mathbf{S}$ and $\mathbf{T}$ are linear operators and that they both have norm 1 on the $L^{\infty}$ spaces where each one is defined. As a consequence, their pre-adjoints $\mathbf{S}^{*}$ and $\mathbf{T}^{*}$, are norm contractions on the corresponding $L^{1}$ spaces. The way things are set up, we have

$$
\begin{aligned}
L_{2}^{\infty}(\mathbb{R}) & =\left\{g+\mathbf{S} g: g \in L^{\infty}(-1,1)\right\}, \\
L_{\langle\beta\rangle}^{\infty}(\mathbb{R}) & =\left\{g+\mathbf{T} g: g \in L^{\infty}(\mathbb{R} \backslash[-\beta, \beta])\right\} .
\end{aligned}
$$

We need the following restriction operators (recall that $\beta>1$ ),

$$
\begin{array}{lll}
\mathbf{R}_{1} & : & L^{\infty}(\mathbb{R} \backslash[-1,1]) \longrightarrow L^{\infty}(\mathbb{R} \backslash[-\beta, \beta]), \\
\mathbf{R}_{2}: & L^{\infty}([-\beta, \beta]) \longrightarrow L^{\infty}([-1,1]), \\
\mathbf{R}_{3}: & L^{\infty}([-\beta, \beta]) \longrightarrow L^{\infty}([-\beta, \beta] \backslash[-1,1]), \\
\mathbf{R}_{4}: & L^{\infty}(\mathbb{R} \backslash[-1,1]) \longrightarrow L^{\infty}([-\beta, \beta] \backslash[-1,1]) .
\end{array}
$$

These operators just restrict the given function to a subset, which make each one a norm contraction. The corresponding pre-adjoints $\mathbf{R}_{i}^{*}$, for $i=1,2,3,4$, act on the corresponding $L^{1}$ spaces, and just extend the given function to a larger set by setting it equal to zero where it was previously undefined. As $\beta>1$, we easily check that $\mathbf{C}_{\beta}^{2}=\mathbf{R}_{2} \mathbf{T} \mathbf{R}_{1} \mathbf{S}$. Taking the pre-adjoint of both sides, we get

$$
\mathbf{P}_{\tau_{\beta}}^{2}=\mathbf{S}^{*} \mathbf{R}_{1}^{*} \mathbf{T}^{*} \mathbf{R}_{2}^{*}
$$

\subsection{The characterization of the pre-annihiliator space $\mathcal{M}_{\beta}^{\perp}$}

We now supply the criterion which characterizes when a given $f \in L^{1}(\mathbb{R})$ belongs to $\mathcal{M}_{\beta}^{\perp}$.

Proposition 6.1. $(1<\beta<+\infty)$ Let $f \in L^{1}(\mathbb{R})$ be written as

$$
f=f_{1}+f_{2}+f_{3}
$$

where $f_{1} \in L^{1}([-1,1]), f_{2} \in L^{1}([-\beta, \beta] \backslash[-1,1])$, and $f_{3} \in L^{1}(\mathbb{R} \backslash[-\beta, \beta])$. Then $f \in \mathcal{M}_{\beta}^{\perp}$ if and only if

$$
\begin{aligned}
\left(\mathbf{I}-\mathbf{P}_{\tau_{\beta}}^{2}\right) f_{1} & =\mathbf{S}^{*}\left(-\mathbf{R}_{4}^{*}+\mathbf{R}_{1}^{*} \mathbf{T}^{*} \mathbf{R}_{3}^{*}\right) f_{2}, \\
f_{3} & =-\mathbf{T}^{*} \mathbf{R}_{2}^{*} f_{1}-\mathbf{T}^{*} \mathbf{R}_{3}^{*} f_{2},
\end{aligned}
$$

where $\mathbf{I}$ is the identity on $L^{1}([-1,1])$.

Proof. In view of the definition (1.3) of $\mathcal{M}_{\beta}^{\perp}$, and the representations (6.3) and (6.4) of $L_{2}^{\infty}(\mathbb{R})$ and $L_{\langle\beta\rangle}^{\infty}(\mathbb{R})$, we have that $f=f_{1}+f_{2}+f_{3}$ is in $\mathcal{M}_{\beta}^{\perp}$ if and only if

$$
\begin{aligned}
& \langle f, g+\mathbf{S} g\rangle_{\mathbb{R}}=\left\langle f_{1}+f_{2}+f_{3}, g+\mathbf{S} g\right\rangle_{\mathbb{R}}=0, \quad g \in L^{\infty}([-1,1]), \\
& \langle f, h+\mathbf{T} h\rangle_{\mathbb{R}}=\left\langle f_{1}+f_{2}+f_{3}, h+\mathbf{T} h\right\rangle_{\mathbb{R}}=0, \quad h \in L^{\infty}(\mathbb{R} \backslash[-\beta, \beta]) .
\end{aligned}
$$

Here, it is assumed that all functions $f_{1}, f_{2}, f_{3}$ are understood to vanish outside their domain of definition. We see that the above equations simplify to

$$
\begin{aligned}
\left\langle f_{1}, g\right\rangle_{[-1,1]}+\left\langle f_{2}+f_{3}, \mathbf{S} g\right\rangle_{\mathbb{R} \backslash[-1,1]} & =0, & & g \in L^{\infty}([-1,1]), \\
\left\langle f_{3}, h\right\rangle_{\mathbb{R} \backslash[-\beta, \beta]}+\left\langle f_{1}+f_{2}, \mathbf{T} h\right\rangle_{[-\beta, \beta]} & =0, & & h \in L^{\infty}(\mathbb{R} \backslash[-\beta, \beta]) .
\end{aligned}
$$


These equations are equivalent to having

$$
\begin{aligned}
& f_{1}=-\mathbf{S}^{*}\left(f_{2}+f_{3}\right), \\
& f_{3}=-\mathbf{T}^{*}\left(f_{1}+f_{2}\right) .
\end{aligned}
$$

A more precise formulation of this is:

$$
\begin{aligned}
& f_{1}=-\mathbf{S}^{*} \mathbf{R}_{4}^{*} f_{2}-\mathbf{S}^{*} \mathbf{R}_{1}^{*} f_{3} ; \\
& f_{3}=-\mathbf{T}^{*} \mathbf{R}_{2}^{*} f_{1}-\mathbf{T}^{*} \mathbf{R}_{3}^{*} f_{2} .
\end{aligned}
$$

We note first that (6.7) is the same as (ii). Next, we substitute (6.7) into (6.6) and take into account (6.5); the result is $(i)$. This completes the proof.

\section{Exterior spectrum of the Perron-Frobenius operator for the Gauss-type map on $[-1,1]$}

\subsection{Purpose of the section}

In this section we will show that $\lambda_{1}=1$ is a simple eigenvalue of $\mathbf{P}_{\tau_{\beta}}$. This corresponds to having a unique absolutely continuous invariant probability measure for $\tau_{\beta}$ with $\beta>1$. We will also prove that $\sigma_{\mathrm{p}}\left(\mathbf{P}_{\tau_{\beta}}\right) \cap \partial \mathbb{D}=\{1\}$. In view of Thorem $\mathbb{C}$ these properties correspond to $\tau_{\beta}$ possessing strong mixing, with exponential decay of correlations (cf. [22], p. 122; also, compare with weak mixing [8], p. 22, p. 29, and [16, p. 203). Another useful reference is [1.

\subsection{The iterates of an interval}

We need the following lemma.

Lemma 7.1. $(1<\beta+\infty)$ Let $J_{0}$ be an nonempty open interval contained in $[-1,1]$. Then, for large enough positive integers $n$, say $n \geq n_{0}$, we have $\left.\tau_{\beta}^{n}\left(J_{0}\right) \supset\right]-1,1[$.

Proof. We begin with the initial observation that if $\left.\tau_{\beta}^{n}\left(J_{0}\right) \supset\right]-1,1\left[\right.$ holds for $n=n_{0}$, then it also holds for all $n \geq n_{0}$, as most of the branches are complete (at most two may be incomplete).

Case I: $\beta$ is an odd integer. Then $\tau_{\beta}$ is "filling", that is, all branches are complete; cf. Subsection 5.3. This case is well-understood, but it helps our presentation to take a look at it again. We recall from Subsection 5.3 that the fundamental intervals are given by (5.4) with $\mathcal{U}$ being the set of all nonzero integers $u$ with $|u| \geq \frac{1}{2}(\beta+1)$. We note that by (5.6), $\tau_{\beta}$ is expansive: as long as an interval $J$ is contained in one of the fundamental intervals $I_{u}, u \in \mathcal{U}$, the image $\tau_{\beta}(J)$ is an interval of length at least $\beta$ times the length of $J$. We observe that if our given interval $J_{0}$ contains one of the fundamental intervals $I_{u}, u \in \mathcal{U}$, then we are finished, because $\left.\tau_{\beta}\left(J_{0}\right) \subset\right]-1,1[$ in this case. There are two other possibilities:

(a) The interval $J_{0}$ is contained in $I_{u}$ for some $u \in \mathcal{U}$ : In this case $\tau_{\beta}\left(J_{0}\right)$ is an interval of length at least $\beta m\left(J_{0}\right)$, by (5.6).

(b) The interval $J_{0}$ has nonempty intersection with two neighboring fundamental intervals $I_{u}, I_{u^{\prime}}$, and $J_{0} \subset \operatorname{clos}\left[I_{u} \cup I_{u^{\prime}}\right]:$ In this case the length of the intersection of $J_{0}$ with one of the two fundamental intervals, say with $I_{u}$, is at least $\frac{1}{2} m\left(J_{0}\right)$. So, we have

$$
m\left(\tau_{\beta}\left(J_{0}\right)\right) \geq m\left(\tau_{\beta}\left(J_{0} \cap I_{u}\right)\right) \geq \frac{\beta}{2} m\left(J_{0}\right)
$$

In particular, $\tau_{\beta}\left(J_{0}\right)$ contains an interval $\tau_{\beta}\left(J_{0} \cap I_{u}\right)$ of length at least $\frac{1}{2} \beta m\left(J_{0}\right)$.

We see that in both cases $(a)-(b)$, the image $\tau_{\beta}\left(J_{0}\right)$ contains an interval $J_{1}$ of length at least $\frac{1}{2} \beta m(J)$. We note that $\frac{1}{2} \beta \geq \frac{3}{2}$ as $\beta>1$ was an odd integer. By running the same argument starting with $J_{1}$ in place of $J_{0}$, we see that unless $J_{1}$ contains a fundamental interval (so that we are finished), we obtain an interval $J_{2}$ contained in $\tau_{\beta}\left(J_{1}\right) \subset \tau_{\beta}^{2}\left(J_{0}\right)$ of length at least $\left(\frac{1}{2} \beta\right)^{2} m\left(J_{0}\right)$. Continuing like this, we find successively intervals $J_{1}, J_{2}, J_{3}, \ldots$ of length $\geq\left(\frac{1}{2} \beta\right)^{l} m\left(J_{0}\right)$ with $J_{l} \subset$ $\tau_{\beta}\left(J_{l-1}\right) \subset \tau_{\beta}^{l}\left(J_{0}\right)$, and we stop only when the interval $J_{l}$ contains a fundamental interval. For a 
big enough $l$ we must stop, at least for the reason that the length of $J_{l}$ will eventually exceed twice the maximum length of a fundamental interval, and for that $l$ we see that $\left.\tau_{\beta}^{l+1}\left(J_{0}\right) \supset\right]-1,1[$.

Case II: $\beta$ is not an odd integer. Then $-1<\{\beta\}_{2}<1$, and with $u_{0}:=\frac{1}{2}\left(\beta-\{\beta\}_{2}\right) \in \mathbb{Z}_{+}, \mathcal{U}$ consists of all integers $u$ with $|u| \geq u_{0}$. We see that $\left.\beta=2 u_{0}+\{\beta\}_{2} \in\right] 2 u_{0}-1,2 u_{0}+1[$. The fundamental intervals $I_{u}$ are given by (5.4) for $u \in \mathcal{U} \backslash\left\{ \pm u_{0}\right\}$, while (cf. (5.3))

$$
\left.I_{u_{0}}=\right] \frac{\beta}{2 u_{0}+1}, 1\left[, \quad I_{-u_{0}}=\right]-1,-\frac{\beta}{2 u_{0}+1}[
$$

On a fundamental interval $I_{u}$, the transformation $\tau_{\beta}$ is given by $x \mapsto 2 u-\beta / x$.

Case II-A: $J_{0}$ is an edge fundamental interval, i.e., $J_{0}=I_{u_{0}}$ or $J_{0}=I_{-u_{0}}$. When $J_{0}=I_{-u_{0}}$, we have

$$
\left.\tau_{\beta}\left(J_{0}\right)=\tau_{\beta}\left(I_{-u_{0}}\right)=\right] \beta-2 u_{0}, 1\left[\supset I_{u_{0}+1}^{2}:=\right] \beta-2 u_{0}, \frac{\beta}{2 u_{0}+1}[.
$$

If $\beta-2 u_{0} \leq \beta /\left(2 u_{0}+3\right)$, we have

$$
\left.\tau_{\beta}\left(I_{-u_{0}}\right)=\right] \beta-2 u_{0}, 1\left[\supset I_{u_{0}+1}^{2} \supset\right] \frac{\beta}{2 u_{0}+3}, \frac{\beta}{2 u_{0}+1}\left[=I_{u_{0}+1},\right.
$$

so that

$$
\left.\tau_{\beta}^{2}\left(I_{-u_{0}}\right) \supset \tau_{\beta}\left(I_{u_{0}+1}\right)=\right]-1,1[\text {. }
$$

It remains to treat the case when $\beta /\left(2 u_{0}+3\right)<\beta-2 u_{0}$, so that $I_{u_{0}+1}^{2} \subset I_{u_{0}+1}$ and in particular, $\beta-2 u_{0} \in I_{u_{0}+1}$. We first claim that there exists a constant $\beta^{\prime}$ with $1<\beta^{\prime} \leq \beta$, which only depends on $\beta$, such that

$$
m\left(\tau_{\beta}(] y, \frac{\beta}{2 u_{0}+1}[)\right) \geq \beta^{\prime} m(] y, 1[), \quad y \in I_{u_{0}+1}^{1}:=\left[\frac{\beta}{2 u_{0}+3}, \beta-2 u_{0}\right] .
$$

Here, it is clear that $I_{u_{0}+1}^{1} \subset I_{u_{0}+1}$, and since $\tau_{\beta}$ is given by $x \mapsto 2 u_{0}+2-\beta / x$ on $I_{u_{0}+1}$, we have that

$$
\left.\tau_{\beta}(] y, \frac{\beta}{2 u_{0}+1}[)=\right] 2 u_{0}+2-\beta / y, 1[,
$$

We realize that the estimate (7.3) is equivalent to having

$$
\beta^{\prime} y+\frac{\beta}{y} \geq 2 u_{0}+1+\beta^{\prime}, \quad y \in I_{u_{0}+1}^{1} .
$$

The function $f(y)=\beta^{\prime} y+\beta / y$ is strictly decreasing in the interval $\left.] 0,1\right]$, so it suffices to check (17.4) at the right endpoint of $I_{u_{0}+1}^{1}$. It is a straightforward exercise to verify that (7.4) holds at $y=\beta-2 u_{0}=\{\beta\}_{2}$ provided that $\beta^{\prime}$ is chosen sufficiently close to 1 ; it helps to observe that $\beta>2$ holds because of $\beta /\left(2 u_{0}+3\right)<\beta-2 u_{0}$. So, (7.4) is valid for $\beta^{\prime}>1$ close enough to 1 . If we put $\left.J_{1}:=\tau_{\beta}\left(I_{-u_{0}}\right)=\right] \beta-2 u_{0}, 1\left[\right.$ and $\left.J_{2}:=\tau_{\beta}\left(I_{u_{0}+1}^{2}\right)=\right] \tau_{\beta}\left(\beta-2 u_{0}\right), 1[$ in accordance with (17.2), then, we have in view of (7.2) and (7.3) that

$$
J_{2} \subset \tau_{\beta}\left(J_{1}\right) \text { and } m\left(J_{2}\right) \geq \beta^{\prime} m\left(J_{1}\right) .
$$

If $\tau_{\beta}\left(\beta-2 u_{0}\right) \leq \beta /\left(2 u_{0}+3\right)$, then $J_{2} \supset I_{u_{0}+1}$ and so $\left.\tau_{\beta}\left(J_{2}\right) \supset\right]-1,1\left[\right.$ and hence $\left.\tau_{\beta}^{3}\left(I_{-u_{0}}\right) \supset\right]-1,1[$. If not, then we rerun the argument and get ever bigger intervals whose right endpoint is 1 , and eventually the interval must contain $I_{u_{0}+1}$, and we are finished. The case $J=I_{u_{0}}$ is analogous and therefore omitted.

Case II-B: $J_{0}$ is a general nonempty open subinterval of $[-1,1]$. We put

$$
x_{0}=\frac{\left(2 u_{0}+1\right) \beta}{2 u_{0}\left(2 u_{0}+1\right)+\beta} .
$$

The point $x_{0}$ belongs to the fundamental interval $I_{u_{0}}$ and has

$$
\tau_{\beta}(] \frac{\beta}{2 u_{0}+1}, x_{0}[)=I_{-u_{0}} \quad \text { and } \quad \tau_{\beta}(]-x_{0},-\frac{\beta}{2 u_{0}+1}[)=I_{u_{0}} .
$$


A little calculation shows that

$$
\tau_{\beta}^{\prime}(x) \geq \frac{\beta}{x_{0}^{2}}>2, \quad x \in\left[-x_{0}, x_{0}\right] \cap \cup\left\{I_{u}: u \in \mathcal{U}\right\} .
$$

Next, put $\beta^{\prime \prime}:=\min \left(\beta, \beta /\left(2 x_{0}^{2}\right)\right)$, so that $\beta^{\prime \prime}>1$. We have a general nonempty subinterval $J_{0}$ of $[-1,1]$, and want to show that $\tau_{\beta}^{n}\left(J_{0}\right)$ covers $]-1,1[$ for some big enough $n$. We do this by showing that the length of $\tau_{\beta}^{n}\left(J_{0}\right)$ must otherwise continue growing geometrically. We observe that if $J_{0}$ contains one of the fundamental intervals $I_{u}, u \in \mathcal{U}$, then $\tau_{\beta}^{n}\left(J_{0}\right) \supset$ ] $-1,1\left[\right.$ with $n=1$ for $|u|>u_{0}$, and with a possibly big $n$ if $|u|=u_{0}$ by Case II-A above. If $J_{0}$ does not contain a fundamental interval, then we are left with the following two possibilities:

(a) The interval $J_{0}$ is contained in a fundamental interval $I_{u}$ for some $u \in \mathcal{U}$. Then $J_{1}:=\tau_{\beta}\left(J_{0}\right)$ is an interval of length at least $\beta^{\prime \prime} m\left(J_{0}\right)$, by (5.6).

(b) The interval $J_{0}$ has nonempty intersection with two neighboring fundamental intervals $I_{u}, I_{u^{\prime}}$, and $J_{0} \subset \operatorname{clos}\left[I_{u} \cup I_{u^{\prime}}\right]$. In this case we have two subcases.

(b1) The interval $J_{0}$ is contained in $\left[-x_{0}, x_{0}\right]$. Then one of the two intervals, say $I_{u}$, meets $J_{0}$ in a subinterval of length at least $\frac{1}{2} m\left(J_{0}\right)$, and we have that $J_{1}:=\tau_{\beta}\left(J_{0} \cap I_{u}\right)$ is an open interval contained in $\tau_{\beta}\left(J_{0}\right)$ of length (cf. (77.6) $)$

$$
m\left(J_{1}\right)=m\left(\tau_{\beta}\left(J_{0} \cap I_{u}\right)\right) \geq \frac{\beta m\left(J_{0}\right)}{2 x_{0}^{2}} \geq \beta^{\prime \prime} m\left(J_{0}\right) .
$$

(b2) The interval $J_{0}$ is not contained in $\left[-x_{0}, x_{0}\right]$. Then we have

$$
J_{0} \cap I_{u_{0}} \supset\left[\frac{\beta}{2 u_{0}+1}, x_{0}\right] \quad \text { or } \quad J_{0} \cap I_{-u_{0}} \supset\left[-x_{0},-\frac{\beta}{2 u_{0}+1}\right],
$$

so by (7.5), we have $\tau_{\beta}(J) \supset I_{-u_{0}}$ or $\tau_{\beta}(J) \supset I_{u_{0}}$.

If (b2) happens, we are finished, because after one iteration of $\tau_{\beta}$ we cover one of the edge fundamental intervals. If $(a)$ or $(b 1)$ take place, then the set $\tau_{\beta}\left(J_{0}\right)$ contains an interval $J_{1}$ of length at least $\beta^{\prime \prime}$ times the length of $J_{0}$. We may then consider $J_{1}$ in place of $J_{0}$, and we gained that $J_{1}$ is longer. Unless we stop, which is because the set contains a fundamental interval, we get a sequence of sets $J_{0}, J_{1}, J_{2}, \ldots$, and their lengths grow geometrically. This is possible only finitely many times, which means that we eventually cover a fundamental interval. The proof is complete.

\subsection{Exterior spectrum of the Perron-Frobenius operator}

For a real-valued function $f$, we use the standard convention to write $f^{+}=\max \{f, 0\}$ and $f^{-}=$ $\max \{-f, 0\}$, so that $f=f^{+}-f^{-}$.

Theorem 7.2. $(1<\beta<+\infty)$ Let $\mathbf{P}_{\tau_{\beta}}$ be the Perron-Frobenius operator associated to $\tau_{\beta}$ acting on $L^{1}([-1,1])$. Then $\lambda_{1}=1$ is a simple eigenvalue of $\mathbf{P}_{\tau_{\beta}}$ and is the only one with modulus one. Moreover, the eigenfunctions for eigenvalue 1 are of the form $\varrho_{0}$, where $c \in \mathbb{C}$ is a constant, and $\varrho_{0} \mathrm{~d} m$ is the unique ergodic $\tau_{\beta}$-invariant absolutely continuous probability measure. Also, $\varrho_{0}>0$ holds almost everywhere.

Proof. To simplify the notation, we write $\mathbf{P}$ in place of $\mathbf{P}_{\tau_{\beta}}$. The transformation $\left.\left.\left.\left.\tau_{\beta}:\right]-1,1\right] \rightarrow\right]-1,1\right]$ is a partially filling $C^{2}$-smooth piecewise monotonic transformation, which meets the conditions $(i)$ [with $m=1$ ] and (ii) of Theorem[C, so that by Remark 5.1 (d), the assertion of Theorem C is valid also for $\tau_{\beta}$. So, we know that that $\lambda_{1}=1$ is an eigenvalue of $\mathbf{P}$ and so there is an eigenfunction $\varrho_{0} \in \mathrm{BV}([-1,1])$ corresponding to it. From (3.5) together with the triangle inequality, we see that $\left|\mathbf{P} \varrho_{0}\right| \leq \mathbf{P}\left|\varrho_{0}\right|$ point-wise. Since $\mathbf{P} \varrho_{0}=\varrho_{0}$, we then have

$$
\int_{[-1,1]}\left|\varrho_{0}\right| \mathrm{d} m=\int_{[-1,1]}\left|\mathbf{P} \varrho_{0}\right| \mathrm{d} m \leq \int_{[-1,1]} \mathbf{P}\left|\varrho_{0}\right| \mathrm{d} m=\int_{[-1,1]}\left|\varrho_{0}\right| \mathrm{d} m .
$$

This means that we must have $|\mathbf{P} \varrho|=\mathbf{P}\left|\varrho_{0}\right|$ almost everywhere on $[-1,1]$, and so in particular, $\mathbf{P}\left|\varrho_{0}\right|=\left|\varrho_{0}\right|$. But then $\left|\varrho_{0}\right|$ is another eigenfunction for $\lambda_{1}=1$. We might as well replace $\varrho_{0}$ by $\left|\varrho_{0}\right|$, 
which amounts to assuming that $\varrho_{0} \geq 0$, and after multiplication by a suitable positive constant we can assume that

We consider the set

$$
\left\langle\varrho_{0}, 1\right\rangle_{[-1,1]}=\int_{[-1,1]} \varrho_{0} \mathrm{~d} m=1
$$

$$
A_{+}:=\left\{x \in[-1,1]: \varrho_{0}(x)>0\right\} .
$$

Using (3.5), we see that $\tau_{\beta}\left(A_{+}\right) \doteq A_{+}$(the dot over the equality sign means that the sets are equal up to Lebesgue null sets). As an element of $\mathrm{BV}([-1,1])$, the function $\varrho_{0}$ can be assumed right-continuous. Then $A_{+}$will contain some non-trivial open interval $I_{0}$. By iteration, we get that $\tau_{\beta}^{n}\left(A_{+}\right) \doteq A_{+}$for $n=1,2,3, \ldots$, so, in particular, $A_{+}$contains $\tau_{\beta}^{n}\left(I_{0}\right)$ (up to null sets). From Lemma 7.1 we know that for a large enough $n, \tau^{n}\left(I_{0}\right)$ covers ] $-1,1\left[\right.$, and so $\varrho_{0}>0$ holds almost everywhere on $[-1,1]$.

Next, we show that the eigenspace for $\lambda_{1}=1$ is one-dimensional. We argue by contradiction, and suppose that that there exists a non-trivial $\eta_{1} \in L^{1}([-1,1])$ so that $\varrho_{0}, \eta_{1}$ are linearly independent, such that $\mathbf{P} \eta_{1}=\eta_{1}$. From Theorem $\mathbb{C}$ we know that $\eta_{1} \in \mathrm{BV}([-1,1])$. We consider the function

$$
f:=\left\{\left\langle\eta_{1}, 1\right\rangle_{[-1,1]}\right\} \varrho_{0}-\eta_{1} \in \mathrm{BV}([-1,1]),
$$

which has $\langle f, 1\rangle_{[-1,1]}=0$, and $\mathbf{P} f=f$. By replacing $\eta_{1}$ by its real or imaginary part (this is possible since $\mathbf{P}$ preserves real-valuedness), we may assume that $\eta_{1}$ is real-valued, so that $f$ is real-valued. We can also assume that $f \in \mathrm{BV}([-1,1])$ is right-continuous. We now write $f=f^{+}-f^{-}$, and observe that $f^{+}, f^{-}$are also right-continuous functions in $\mathrm{BV}([-1,1])$. Unless one of the functions $f^{+}, f^{-}$vanishes almost everywhere, both $f^{+}, f^{-}$must be positive on some open intervals $I_{+}, I_{-}$, respectively. In view of (3.5), we then have that for $n=1,2,3, \ldots$, the functions $\mathbf{P}^{n} f^{+}, \mathbf{P}^{n} f^{-}$are positive almost everywhere on the sets $\tau^{n}\left(I_{+}\right), \tau^{n}\left(I_{-}\right)$, respectively. This means that Lemma 7.1 entails that there exists a positive integer $n_{0}$ such that $\mathbf{P}^{n_{0}} f^{+}, \mathbf{P}^{n_{0}} f^{-}$are both positive almost everywhere on $[-1,1]$. Since $\mathbf{P}^{n} f=f$ for all $n=1,2,3, \ldots$, we must then have

$$
\|f\|_{L^{1}}=\left\|\mathbf{P}^{n_{0}} f\right\|_{L^{1}}=\left\|\mathbf{P}^{n_{0}} f^{+}-\mathbf{P}^{n_{0}} f^{-}\right\|_{L^{1}}<\left\|\mathbf{P}^{n_{0}} f^{+}+\mathbf{P}^{n_{0}} f^{-}\right\|_{L^{1}}=\|f\|_{L^{1}},
$$

where the $L^{1}$ norm is with respect to the interval $[-1,1]$. This contradiction shows that at least one of $f^{+}, f^{-}$must be identically zero (almost everywhere). But as $\langle f, 1\rangle_{[-1,1]}=0$, both $f^{+}, f^{-}$ must then be the 0 function. This does not agree with our assumption that $\varrho_{0}, \eta_{1}$ should be linearly independent. We conclude that that $\lambda_{1}=1$ is a simple eigenvalue (i.e., it has a one-dimensional eigenspace).

Next, we turn to the assertion that $\varrho_{0} \mathrm{~d} m$ is the unique ergodic absolutely continuous probability measure. This is a consequence of Proposition A.2.5 in [16], since we know that $\varrho_{0} \mathrm{~d} m$ is an absolutely continuous measure with $\varrho_{0}>0$ almost everywhere, and $\mathbf{P} \varrho_{0}=\varrho_{0}$.

Finally, we show that $\mathbf{P}$ has no other eigenvalues than 1 on the unit circle $\mathbb{T}$. We let $\lambda$, with $|\lambda|=1$ and $\lambda \neq 1$, be an eigenvalue of $\mathbf{P} ; \eta_{2} \in L^{1}([-1,1])$ is the non-trivial eigenfunction corresponding to $\lambda$, which we may normalize: $\left\|\eta_{2}\right\|_{L^{1}}=1$. From (3.5) together with the triangle inequality, we have $\left|\mathbf{P} \eta_{2}\right| \leq \mathbf{P}\left|\eta_{2}\right|$ point-wise, and so, since $\mathbf{P} \eta_{2}=\lambda \eta_{2}$,

$$
\int_{[-1,1]}\left|\eta_{2}\right| \mathrm{d} m=\int_{[-1,1]}\left|\lambda \eta_{2}\right| \mathrm{d} m=\int_{[-1,1]}\left|\mathbf{P} \eta_{2}\right| \mathrm{d} m \leq \int_{[-1,1]} \mathbf{P}\left|\eta_{2}\right| \mathrm{d} m=\int_{[-1,1]}\left|\eta_{2}\right| \mathrm{d} m .
$$

This means that we must have $\left|\mathbf{P} \eta_{2}\right|=\mathbf{P}\left|\eta_{2}\right|$ almost everywhere on $[-1,1]$, and, consequently, $\mathbf{P}\left|\eta_{2}\right|=\left|\eta_{2}\right|$. But then $\left|\eta_{2}\right|=\varrho_{0}$, as the eigenspace for $\lambda_{1}=1$ was one-dimensional and spanned by $\varrho_{0}$. We write $\eta_{2}=\chi \varrho_{0}$, where the function $\chi \in L^{\infty}([-1,1])$ has $|\chi|=1$ almost everywhere. When we take another look at the argument we just did involving equality in the triangle inequality, we realize that $\chi$ must have the property

$$
\chi\left(\tau_{\beta}(x)\right)=\bar{\lambda} \chi(x), \quad x \in[-1,1]
$$

in the almost-everywhere sense. By iteration, we get that

$$
\chi\left(\tau_{\beta}^{n}(x)\right)=\bar{\lambda}^{n} \chi(x), \quad x \in[-1,1], n=1,2,3, \ldots
$$


We pick a point $x_{0}$ where $\rho_{0}\left(x_{0}\right)>0$, and by right-continuity there exists a non-empty (short) interval $] x_{0}, x_{1}$ [ where $\rho_{0}, \eta_{2}$ are both very close to the value at $x_{0}$, so that $\chi$ is close to its value at $x_{0}$ as well: say, for some small $\epsilon>0$,

$$
\left.\left|\chi(x)-\chi\left(x_{0}\right)\right|<\epsilon, \quad x \in\right] x_{0}, x_{1}[.
$$

Next, let $n$ be such that $\left.\tau_{\beta}^{n}(] x_{0}, x_{1}[) \supset\right]-1,1[$. Then, by (7.8),

$$
\left.\left|\chi\left(\tau_{\beta}^{n}(x)\right)-\lambda^{n} \chi\left(x_{0}\right)\right|<\epsilon, \quad x \in\right] x_{0}, x_{1}[,
$$

so that

$$
\left.\left|\chi(y)-\lambda^{n} \chi\left(x_{0}\right)\right|<\epsilon, \quad y \in\right]-1,1[.
$$

This means that $\chi$ is within a distance $\epsilon$ from a constant function. As $\epsilon$ can be made as small as we like, the only possibility is that $\chi$ is equal to a constant. But then (7.8) is impossible unless $\lambda=1$, contrary to assumption. The proof is complete.

Remark 7.3. $(1<\beta<+\infty)$ A dynamical system $(I, \mathfrak{S}, \mu, \tau)$, where $\mu$ is finite and invariant under $\tau$, is said to be exact when we have

$$
\lim _{n \rightarrow+\infty} \mu\left(\tau^{n}(A)\right)=\mu(I), \quad A \in \mathfrak{S} .
$$

It is known that when $(I, \mathfrak{S}, \mu, \tau)$ is exact, the $\tau$ possesses strong mixing [22], p. 125. If $\varrho_{0}$ stands for the density of the unique absolutely continuous $\tau_{\beta}$-invariant probability measure, then $\left([-1,1], \mathfrak{B}_{[-1,1]}, \varrho_{0} \mathrm{~d} m, \tau_{\beta}\right)$ is exact. In particular,

$$
\lim _{n \rightarrow+\infty} m\left(\tau_{\beta}^{n}(A)\right)=m([-1,1]), \quad A \in \mathfrak{B}_{[-1,1]} .
$$

This can be obtained from Lemma 7.1 directly, by localizing around a point of $A$ with density 1 and using the distortion control available from the control on the second derivative; cf. [14. This suggests another (shorter) way to obtain the assertion of Theorem 7.2. We get that $\tau_{\beta}$ possesses strong mixing from the exactness, and then there can only be the eigenvalue 1 and it must be simple.

\section{Proofs of the main results}

\subsection{Proof of Theorem 1.3}

To prove Theorem 1.3 , we will need to consider the space $\mathrm{BV}([-\beta, \beta] \backslash[-1,1])$ of complex-valued integrable functions defined on $[-\beta,-1] \cup[1, \beta]$ whose restrictions to $[-\beta,-1]$ belong to $\mathrm{BV}([-\beta,-1])$ and whose restrictions to $[1, \beta]$ belong to $\mathrm{BV}([1, \beta])$.

Lemma 8.1. $(1<\beta<+\infty)$ The operator $-\mathbf{S}^{*} \mathbf{R}_{4}^{*}+\mathbf{S}^{*} \mathbf{R}_{1}^{*} \mathbf{T}^{*} \mathbf{R}_{3}^{*}$ maps $\operatorname{BV}([-\beta, \beta] \backslash[-1,1])$ into $\mathrm{BV}([-1,1])$.

Proof. Let $f_{2} \in \mathrm{BV}([-\beta, \beta] \backslash[-1,1])$. The operator $\mathbf{S}^{*}: L^{1}(\mathbb{R} \backslash[-1,1]) \rightarrow L^{1}([-1,1])$ is given by

$$
\mathbf{S}^{*} h(x)=\sum_{k \in \mathbb{Z}^{\times}} h(x+2 k), \quad x \in[-1,1] .
$$

As

$$
\mathbf{R}_{4}^{*}: L^{1}([-\beta, \beta] \backslash[-1,1]) \rightarrow L^{1}(\mathbb{R} \backslash[-1,1])
$$

extends the function to vanish where it was previously undefined, the function $\mathbf{S}^{*} \mathbf{R}_{4}^{*} f_{2}$ is just a finite sum of functions of bounded variation, so that Then we have

$$
\mathbf{S}^{*} \mathbf{R}_{4}^{*} f_{2} \in \mathrm{BV}([-1,1])
$$

On the other hand, we can easily check that $\tilde{\mathbf{C}}^{2}=\mathbf{T R}_{1} \mathbf{S R}_{2}$, where $\tilde{\mathbf{C}}=\mathbf{C}_{\tilde{\tau}_{\beta}}$ is the Koopman operator associated to the transformation $\tilde{\tau}_{\beta}:[-\beta, \beta] \rightarrow[-\beta, \beta]$ given by $\tilde{\tau}_{\beta}(0)=0$ and

$$
\tilde{\tau}_{\beta}(x)=\{-\beta / x\}_{2}, \quad x \in[-\beta, \beta] \backslash\{0\} .
$$


If $\tilde{\mathbf{P}}=\mathbf{P}_{\tilde{\tau}_{\beta}}: L^{1}([-\beta, \beta]) \rightarrow L^{1}([-\beta, \beta])$ is the corresponding Perron-Frobenius operator, whose adjoint is $\tilde{\mathbf{C}}$, we find that

$$
\tilde{\mathbf{P}}^{2}=\mathbf{R}_{2}^{*} \mathbf{S}^{*} \mathbf{R}_{1}^{*} \mathbf{T}^{*}
$$

We easily check that $\tilde{\tau}_{\beta}$ satisfies conditions $(i)$ (with $m=2$ ) and (ii) of Theorem. However, the mapping is only "partially filling". But in view of Remark 5.1, Theorem C remains valid nevertheless. In particular, we have that $\tilde{\mathbf{P}}$ transforms $\mathrm{BV}([-\beta, \beta])$ into itself. Since $\mathbf{R}_{3}^{*}$ and $\mathbf{R}_{2}^{*}$ are just extension by zero operators, it follows from (8.2) that

$$
\mathbf{S}^{*} \mathbf{R}_{1}^{*} \mathbf{T}^{*} \mathbf{R}_{3}^{*} f_{2} \in \mathrm{BV}([-1,1]) \text {. }
$$

Adding up, we get from (8.1) and (8.3) that

$$
-\mathbf{S}^{*} \mathbf{R}_{4}^{*} f_{2}+\mathbf{S}^{*} \mathbf{R}_{1}^{*} \mathbf{T}^{*} \mathbf{R}_{3}^{*} f_{2} \in \mathrm{BV}([-1,1])
$$

for every $f_{2} \in \mathrm{BV}([-\beta, \beta] \backslash[-1,1])$. The proof is complete.

We have now developed the tools needed to obtain Theorem 1.3. Actually, we formulate a more precise result.

Theorem 8.2. $(1<\beta<+\infty)$ There exists a bounded operator $\mathbf{E}: \operatorname{BV}([-\beta, \beta] \backslash[-1,1]) \rightarrow L^{1}(\mathbb{R})$ with the following properties:

(i) $\mathbf{E}$ is an extension operator, in the sense that $\mathbf{E} f(x)=f(x)$ almost everywhere on $[-\beta, \beta] \backslash[-1,1]$ for all $f \in \mathrm{BV}([-\beta, \beta] \backslash[-1,1])$.

(ii) The range of $\mathbf{E}$ is infinite-dimensional, and contained in $\mathcal{M}_{\beta}^{\perp}$.

Proof. To simplify the notation, we write $\mathbf{P}$ in place of $\mathbf{P}_{\tau_{\beta}}$. By Theorem $\mathbf{C}$ (valid by Remark 5.1 $(d)$ ) together with Theorem [7.2, we have the following representation for the iterates of $\mathbf{P}$,

$$
\mathbf{P}^{n} h=\left\{\langle h, 1\rangle_{[-1,1]}\right\} \varrho_{0}+\mathbf{Z}^{n} h, \quad h \in L^{1}([-1,1]), \quad n=1,2,3, \ldots,
$$

where we write $\mathbf{Z}$ in place of $\mathbf{Z}_{\tau_{\beta}}$. Here, $\varrho_{0} \geq 0$ is the density for the absolutely continuous $\tau_{\beta}$-invariant probability measure on $[-1,1]$; it has $\varrho_{0} \in \mathrm{BV}([-1,1])$. The operator $\mathbf{Z}$ acts on $\mathrm{BV}([-1,1])$ and its spectral radius is $<1$. By applying (8.4) to $h=\varrho_{0}$, it is evident that $\mathbf{Z} \varrho_{0}=0$. Next, if we note that

$$
\left\langle\mathbf{P}^{n} h, 1\right\rangle_{[-1,1]}=\langle h, 1\rangle_{[-1,1]}, \quad n=1,2,3, \ldots,
$$

which is one of the standard properties of Perron-Frobenius operators (e.g., we can use that 1 is invariant under the Koopman operator), we also get that

$$
\left\langle\mathbf{Z}^{n} h, 1\right\rangle_{[-1,1]}=0, \quad h \in L^{1}([-1,1]), \quad n=1,2,3, \ldots
$$

Let us take an arbitrary element $f \in \operatorname{BV}([-\beta, \beta] \backslash[-1,1])$. From Lemma 8.1 above, we know that

$$
-\mathbf{S}^{*} \mathbf{R}_{4}^{*} f+\mathbf{S}^{*} \mathbf{R}_{1}^{*} \mathbf{T}^{*} \mathbf{R}_{3}^{*} f \in \mathrm{BV}([-1,1]) .
$$

Since $\mathbf{Z}$ has spectral radius $<1$ on $\mathrm{BV}([-1,1])$, the Neumann series

$$
\left(\mathbf{I}-\mathbf{Z}^{2}\right)^{-1}=\mathbf{I}+\mathbf{Z}^{2}+\mathbf{Z}^{4}+\ldots
$$

converges to a bounded operator on $\mathrm{BV}([-1,1])$, and we may put

$$
\mathbf{E}_{1} f:=\left(\mathbf{I}-\mathbf{Z}^{2}\right)^{-1} \mathbf{S}^{*}\left\{-\mathbf{R}_{4}^{*} f+\mathbf{R}_{1}^{*} \mathbf{T}^{*} \mathbf{R}_{3}^{*} f\right\} \in \mathrm{BV}([-1,1]) .
$$

We observe that

$$
\left\langle-\mathbf{S}^{*} \mathbf{R}_{4}^{*} f+\mathbf{S}^{*} \mathbf{R}_{1}^{*} \mathbf{T}^{*} \mathbf{R}_{3}^{*} f, 1\right\rangle_{[-1,1]}=\langle f, 1\rangle_{[-\beta, \beta] \backslash[-1,1]}-\langle f, 1\rangle_{[-\beta, \beta] \backslash[-1,1]}=0,
$$

and so, by (8.5) and (8.6),

$$
\left\langle\mathbf{E}_{1} f, 1\right\rangle_{[-1,1]}=\left\langle\left(\mathbf{I}-\mathbf{Z}^{2}\right) \mathbf{E}_{1} f, 1\right\rangle_{[-1,1]}=0 .
$$

Finally, we put

$$
\mathbf{E}_{3} f:=-\mathbf{T}^{*}\left(\mathbf{R}_{2}^{*} \mathbf{E}_{1}+\mathbf{R}_{3}^{*}\right) f \in L^{1}(\mathbb{R} \backslash[-\beta, \beta]) .
$$


We define the operator $\mathbf{E}$ to be the mapping

$$
\mathbf{E}: \mathrm{BV}([-\beta, \beta] \backslash[-1,1]) \rightarrow L^{1}(\mathbb{R}), \quad f \mapsto f+\mathbf{E}_{1} f+\mathbf{E}_{3} f,
$$

with the understanding that each of the functions $f, \mathbf{E}_{1} f, \mathbf{E}_{2} f$ is extended to $\mathbb{R}$ by putting it equal to 0 where it was previously undefined. Then $\mathbf{E}$ is clearly bounded and linear, and has the property $(i)$. In view of (8.7) and (8.4), we have $\mathbf{P}^{n} \mathbf{E}_{1} f=\mathbf{Z}^{n} \mathbf{E}_{1} f$ for $n=1,2,3, \ldots$. This means that in condition $(i)$ of Proposition 6.1, we may replace $\mathbf{P}^{2}$ by $\mathbf{Z}^{2}$, and we just obtain the condition Proposition 6.1 (i) rather immediately from (8.6). The condition Proposition 6.1 (ii) is immediate from (8.8). By Proposition 6.1, we have that

$$
\operatorname{im} \mathbf{E} \subset \mathcal{M}_{\beta}^{\perp},
$$

which proves (ii), since im $\mathbf{E}$ - the range of $\mathbf{E}$ - must be infinite-dimensional (the restriction to $[-\beta, \beta] \backslash[-1,1]$ of the range is infinite-dimensional, being the space of all functions of bounded variation). This completes the proof of Theorem 1.3 .

Proof of Theorem 1.3. This is immediate from Theorem 8.2 (ii).

The next theorem shows that the range of $\mathbf{E}$ we constructed in in the proof of Theorem 1.3 is a subspace of the weighted space $L^{2}(\mathbb{R}, \omega)$, where $\omega(x):=1+x^{2}$, with finite norm

$$
\|f\|_{L^{2}(\mathbb{R}, \omega)}^{2}:=\int_{\mathbb{R}}|f|^{2} \omega \mathrm{d} m<+\infty .
$$

Proposition 8.3. The range of $\mathbf{E}$ is contained in $L^{2}(\mathbb{R}, \omega)$.

Proof. Let $f \in \mathrm{BV}([-\beta, \beta] \backslash[-1,1])$. Following the proof of Theorem 8.2 , we see that $\mathbf{E}_{1} f \in$ $\mathrm{BV}([-1,1])$, so that the restriction of $\mathbf{E} f$ to the interval $[-\beta, \beta]$ has bounded variation. In particular, $\mathbf{E} f$ is bounded on $[-\beta, \beta]$, so we just to estimate the weighted $L^{2}$-norm integral on $\mathbb{R} \backslash[-\beta, \beta]$. The restriction of $\mathbf{E} f$ to $\mathbb{R} \backslash[-\beta, \beta]$ equals $\mathbf{E}_{3} f$, given by (8.6), which we understand as $\mathbf{E}_{3} f=-\mathbf{T}^{*} h$. The operator $\mathbf{T}^{*}: L^{1}([-\beta, \beta]) \rightarrow L^{1}(\mathbb{R} \backslash[-\beta, \beta])$ is given explicitly by

$$
\mathbf{T}^{*} h(x)=\sum_{j \in \mathbb{Z}^{\times}} \frac{\beta^{2}}{(\beta+2 j x)^{2}} h\left(\frac{\beta x}{\beta+2 j x}\right),
$$

with the understanding that $h$ is extended to vanish off $[-\beta, \beta]$. We write

$$
h_{j}(x)=h\left(\frac{\beta x}{\beta+2 j x}\right) \frac{\beta^{2}}{(\beta+2 j x)^{2}}, \quad x \in \mathbb{R} \backslash[-\beta, \beta] .
$$

As $h$ is bounded, it is clear from (8.9) that $\mathbf{E}_{3} f$ is bounded on $\mathbb{R} \backslash[-\beta, \beta]$. Since $\mathbf{E}_{3} f$ is also summable, we must have

Hence, it is enough to show that

$$
\int_{\mathbb{R} \backslash[-\beta, \beta]}\left|\mathbf{E}_{3} f(x)\right|^{2} \mathrm{~d} x<+\infty .
$$

$$
\int_{\mathbb{R} \backslash[-\beta, \beta]}\left|\mathbf{E}_{3} f(x)\right|^{2} x^{2} \mathrm{~d} x=\int_{\mathbb{R} \backslash[-\beta, \beta]}\left|\sum_{j \in \mathbb{Z}^{\times}} h_{j}(x)\right|^{2} x^{2} \mathrm{~d} x<+\infty .
$$

A straightforward computation shows that

$$
\int_{\mathbb{R} \backslash[-\beta, \beta]}\left|h_{j}(x)\right|^{2} x^{2} \mathrm{~d} x=\int_{\frac{\beta}{2 j+1}}^{\frac{\beta}{2 j-1}}|h(x)|^{2} x^{2} \mathrm{~d} x \leq\|h\|_{L^{\infty}([-\beta, \beta])}^{2} \int_{\frac{\beta}{2 j+1}}^{\frac{\beta}{2 j-1}} x^{2} \mathrm{~d} x \leq \frac{\beta^{2}}{j^{4}}\|h\|_{L^{\infty}([-\beta, \beta])}^{2} .
$$

As a consequence, we obtain

$$
\sum_{j \in \mathbb{Z}^{\times}}\left\{\int_{\mathbb{R} \backslash[-\beta, \beta]}\left|h_{j}(x)\right|^{2} x^{2} \mathrm{~d} x\right\}^{\frac{1}{2}}<+\infty
$$

which entails (8.10). The proof is complete. 
Remark 8.4. The range of the operator $\mathbf{E}$ is a proper subspace of $\mathcal{M}_{\beta}^{\perp}$, even if we consider the closure of the range. Actually, if in the context of Proposition 6.1] we plug in $f_{1}:=\varrho_{0}$ (notation as in Theorem (7.2) and $f_{2}:=0$, and put $f_{3}:=-\mathbf{T}^{*} \mathbf{R}_{2} \varrho_{0}$, we obtain a function $\psi_{0}:=f_{1}+f_{2}+f_{3}=$ $\varrho_{0}-\mathbf{T}^{*} \mathbf{R}_{2} \varrho_{0}$ which is in the annihilator $\mathcal{M}_{\beta}^{\perp}$, but $\psi_{0}$ is not in the closure of the range of $\mathbf{E}$. So a natural question is whether

$$
\operatorname{span}\left\{\psi_{0}\right\} \oplus \cos [\operatorname{im} \mathbf{E}]=\mathcal{M}_{\beta}^{\perp}
$$

holds. This would be quite reasonable from the point of view of the proof of Proposition 6.1.

\subsection{Proof of Theorem 1.4}

We turn to the proof of Theorem 1.4. We know from [13] that $\mathcal{N}_{\beta}^{\perp}$ is one-dimensional for $\beta=2$. We shall write $\beta=2 \gamma$, and suppose that $\gamma>1$. We need the operators

$$
\mathbf{S}_{+}: L^{\infty}([0,1]) \rightarrow L^{\infty}\left(\left[1,+\infty[), \quad \mathbf{S}_{+} g(x):=g\left(\{x\}_{1}\right)\right.\right.
$$

and

$$
\mathbf{T}_{+}: L^{\infty}\left(\left[\gamma,+\infty[) \rightarrow L^{\infty}([0, \gamma]), \quad \mathbf{T}_{+} g(x):=g\left(\frac{\gamma}{\{\gamma / x\}_{1}}\right) .\right.\right.
$$

Their pre-adjoints map contractively

$$
\mathbf{S}_{+}^{*}: L^{1}\left(\left[1,+\infty[) \rightarrow L^{1}([0,1]), \quad \mathbf{T}_{+}^{*}: L^{1}([0, \gamma]) \rightarrow L^{1}([\gamma,+\infty[) .\right.\right.
$$

We need the following restriction operators:

$$
\begin{array}{ll}
\mathbf{R}_{5}: & L^{\infty}\left(\left[1,+\infty[) \longrightarrow L^{\infty}([\gamma,+\infty[),\right.\right. \\
\mathbf{R}_{6}: & L^{\infty}([0, \gamma]) \longrightarrow L^{\infty}([0,1]), \\
\mathbf{R}_{7}: & L^{\infty}([0, \gamma]) \longrightarrow L^{\infty}([1, \gamma]), \\
\mathbf{R}_{8}: & L^{\infty}\left(\left[1,+\infty[) \longrightarrow L^{\infty}([1, \gamma]) .\right.\right.
\end{array}
$$

and their pre-adjoints $\mathbf{R}_{5}^{*}, \mathbf{R}_{6}^{*}, \mathbf{R}_{7}^{*}, \mathbf{R}_{8}^{*}$, which act on the corresponding $L^{1}$-spaces. We let $\mathbf{P}_{+}:=\mathbf{P}_{\theta_{\gamma}}$ denote the Perron-Frobenius operator associated to the Gauss-type transformation $\theta_{\gamma}:[0,1[\rightarrow$ $\left[0,1\left[\right.\right.$, where $\theta_{\gamma}(0):=0$ and $\theta_{\gamma}(x):=\{\gamma / x\}_{1}$ for $\left.x \in\right] 0,1[$; cf. Section 4 . The analogue of (6.5) in this context is

$$
\mathbf{P}_{+}^{2}=\mathbf{S}_{+}^{*} \mathbf{R}_{5}^{*} \mathbf{T}_{+}^{*} \mathbf{R}_{6}^{*} .
$$

We also have an analogue of Proposition 6.1.

Proposition 8.5. $(1<\gamma<+\infty)$ Let $f \in L^{1}([0,+\infty[)$ be written as

$$
f=f_{1}+f_{2}+f_{3}
$$

where $f_{1} \in L^{1}\left(\left[0,1[), f_{2} \in L^{1}\left(\left[1, \gamma[)\right.\right.\right.\right.$, and $f_{3} \in L^{1}\left(\left[\gamma,+\infty[)\right.\right.$. Then $f \in \mathcal{N}_{2 \gamma}^{\perp}$ if and only if

$$
\begin{aligned}
\left(\mathbf{I}-\mathbf{P}_{+}^{2}\right) f_{1} & =\mathbf{S}_{+}^{*}\left(-\mathbf{R}_{8}^{*}+\mathbf{R}_{5}^{*} \mathbf{T}_{+}^{*} \mathbf{R}_{7}^{*}\right) f_{2}, \\
f_{3} & =-\mathbf{T}_{+}^{*} \mathbf{R}_{6}^{*} f_{1}-\mathbf{T}_{+}^{*} \mathbf{R}_{7}^{*} f_{2},
\end{aligned}
$$

where $\mathbf{I}$ is the identity on $L^{1}([0,1])$.

The proof is completely analogous to that of Proposition 6.1, and we omit it. Since $\gamma>1$, the transformation $\theta_{\gamma}$ is uniformly expansive, and if we analyze its spectral properties on $\mathrm{BV}([0,1])$, we see that $\mathbf{P}_{+}$has a spectral gap. More precisely, in the context of Theorem $\mathbf{C}$ [valid by Remark $5.1(d)]$, we can show that $\sigma\left(\mathbf{P}_{+}\right) \cap \mathbb{T}=\{1\}$ and that the eigenvalue 1 is simple. This leads to the following assertion, analogous to Theorem 8.2 Again, we omit the proof.

Theorem 8.6. $(1<\gamma<+\infty)$ There exists a bounded operator $\mathbf{E}_{+}: \mathrm{BV}([1, \gamma]) \rightarrow L^{1}([0,+\infty[)$ with the following properties:

(i) $\mathbf{E}_{+}$is an extension operator, in the sense that $\mathbf{E}_{+} f(x)=f(x)$ almost everywhere on $[1, \gamma]$ for all $f \in \mathrm{BV}([1, \gamma])$.

(ii) The range of $\mathbf{E}_{+}$is infinite-dimensional, and contained in $\mathcal{N}_{2 \gamma}^{\perp}$.

Proof of Theorem 1.4. This is immediate from Theorem 8.6 (ii). 


\section{Final remarks}

\subsection{A related problem in the Hardy space of the unit disk}

An algebra of inner functions in the Hardy spaces $H^{p}$ of the unit disk was considered by Matheson and Stessin in 21. This algebra depends on a parameter $\beta>0$, and can be assumed to be

$$
\mathcal{A}_{\beta}=\operatorname{span}\left\{\mathrm{e}^{-\pi m \frac{1-z}{1+z}} \mathrm{e}^{-\pi n \beta \frac{1+z}{1-z}}: m, n=0,1,2, \ldots\right\},
$$

where the span is in the sense of finite linear combinations. The main result [21] asserts that for any finite $p, \mathcal{A}_{\beta}$ is dense in $H^{p}$ for $\beta<1$, while it fails to be dense for $\beta>1$. It is natural to also consider the (smaller) space

$$
\mathcal{S}_{\beta}=\operatorname{span}\left\{\mathrm{e}^{-\pi m \frac{1-z}{1+z}}, \mathrm{e}^{-\pi n \beta \frac{1+z}{1-z}}: m, n=0,1,2, \ldots\right\} .
$$

For $n=0,1,2, \ldots$, , let $\varphi^{n}$ denote the function

$$
\varphi^{n}(z):=\mathrm{e}^{-\pi n \frac{1-z}{1+z}} \mathrm{e}^{-\pi n \beta \frac{1+z}{1-z}}, \quad z \in \mathbb{D} .
$$

Then we clearly have the decomposition

$$
\mathcal{A}_{\beta}=\mathcal{S}_{\beta}+\varphi \mathcal{S}_{\beta}+\varphi^{2} \mathcal{S}_{\beta}+\ldots,
$$

in the sense of finite sums. As a consequence of the main result in [12, Theorem $\mathrm{A}$, we know that $\mathcal{S}_{1}$ is dense in the weak-star topology of BMOA, and hence in the norm topology in $H^{p}$ for all finite $p$. It would appear rather plausible that the codimension of the $H^{p}$-closure of $\mathcal{A}_{\beta}$ might be finite $\beta>1$, as there are only finitely many points in the unit disk which are not separated by the generating inner functions. However, so far, we cannot provide an answer to this question. With the aid of Theorem [1.3 we can however prove that the $H^{2}$-closure of the space $\mathcal{S}_{\beta}$ has infinite codimension for $\beta>1$. An outline of the argument is provided below.

We assume $\beta>1$, and pick an arbitrary natural number $N \geq 1$. By Theorem 8.2 , we can pick linearly independent elements $f_{1}, \ldots, f_{N} \in \mathcal{M}_{\beta}^{\perp}$ in the range of $\mathbf{E}$, which all vanish on some proper subinterval of $[1, \beta]$, say on $\left[1, \beta^{\prime}\right]$, where $1<\beta^{\prime}<\beta$. We define linearly independent functions $\tilde{f}_{j}$, $j=1, \ldots, N$, on the unit circle $\mathbb{T}$ as follows:

$$
\frac{1}{1-\mathrm{i} x} \tilde{f}_{j}\left(\frac{1+\mathrm{i} x}{1-\mathrm{i} x}\right)=(1+i x) f_{j}(x), \quad j=1, \ldots, N .
$$

Next, by Proposition 8.3 , we have that the functions $\tilde{f}_{j}$ belong to $L^{2}(\mathbb{T})$ and that they all vanish on a certain $\operatorname{arc}$ of $\mathbb{T}$. Let $\mathbf{Q}: L^{2}(\mathbb{T}) \rightarrow H^{2}$ denote the orthogonal (Szegö) projection. We claim that the projected functions $\mathbf{Q} \tilde{f}_{j}, j=1, \ldots, N$, are linearly independent. Indeed, a relation of the form

$$
\sum_{j=1}^{N} c_{j} \mathbf{Q} \tilde{f}_{j}=0, \quad c_{j} \in \mathbb{C},
$$

implies that the function $\tilde{f}_{\text {sum }}:=\sum_{j=1}^{N} c_{j} \tilde{f}_{j}$ belongs to $L^{2}(\mathbb{T}) \ominus H^{2}=\operatorname{conj} H_{0}^{2}$, where "conj" means complex conjugation, and $H_{0}^{2}$ is the subspace of $H^{2}$ of functions that vanish at the origin. Now the function $\tilde{f}_{\text {sum }}$ is in conj $H_{0}^{2}$ and vanishes along an $\operatorname{arc}$ of the circle $\mathbb{T}$, so by e.g. Privalov's theorem, $\tilde{f}_{\text {sum }}=0$ on all of $\mathbb{T}$. From this and the linear independence of the functions $\tilde{f}_{1}, \ldots, \tilde{f}_{N}$, we obtain $c_{j}=0$ for all $j=1, \ldots, N$. So, the projected functions $\mathbf{Q} \tilde{f}_{j}, j=1, \ldots, N$, are linearly independent, as claimed. Finally, we claim that $\mathbf{Q} \tilde{f}_{j}, j=1, \ldots, N$, belong to the orthocomplement of $\mathcal{S}_{\beta}$ in $H^{2}$. If $(\cdot, \cdot)_{H^{2}}$ denotes the sesquilinear inner product of $H^{2}$, we calculate that for $m=0,1,2, \ldots$,

$$
\begin{aligned}
\left(\mathbf{Q} \tilde{f}_{j}, \mathrm{e}^{-\pi m \frac{1-z}{1+z}}\right)_{H^{2}}=\frac{1}{2 \pi} \int_{\mathbb{T}} \mathbf{Q} \tilde{f}_{j}(\zeta) \operatorname{conj}\left(\mathrm{e}^{-\pi m \frac{1-\zeta}{1+\zeta}}\right)|\mathrm{d} \zeta| \\
\quad=\frac{1}{2 \pi} \int_{\mathbb{T}} \tilde{f}_{j}(\zeta) \operatorname{conj}\left(\mathrm{e}^{-\pi m \frac{1-\zeta}{1+\zeta}}\right)|\mathrm{d} \zeta|=\frac{1}{\pi} \int_{\mathbb{R}} f_{j}(x) \mathrm{e}^{\mathrm{i} \pi m x} \mathrm{~d} x=0,
\end{aligned}
$$


where the last equality uses that $f_{j} \in \mathcal{M}_{\beta}^{\perp}$. In the analogous fashion, we get that for $n=0,1,2, \ldots$,

$$
\begin{aligned}
\left(\mathbf{Q} \tilde{f}_{j}, \mathrm{e}^{-\pi \beta n \frac{1+z}{1-z}}\right)_{H^{2}}=\frac{1}{2 \pi} \int_{\mathbb{T}} \mathbf{Q} \tilde{f}_{j}(\zeta) \operatorname{conj}\left(\mathrm{e}^{-\pi \beta n \frac{1+\zeta}{1-\zeta}}\right)|\mathrm{d} \zeta| \\
\quad=\frac{1}{2 \pi} \int_{\mathbb{T}} \tilde{f}_{j}(\zeta) \operatorname{conj}\left(\mathrm{e}^{-\pi \beta n \frac{1+\zeta}{1-\zeta}}\right)|\mathrm{d} \zeta|=\frac{1}{\pi} \int_{\mathbb{R}} f_{j}(x) \mathrm{e}^{-\mathrm{i} \pi \beta n / x} \mathrm{~d} x=0,
\end{aligned}
$$

where again we use that $f_{j} \in \mathcal{M}_{\beta}^{\perp}$. It follows that the codimension of the $H^{2}$-closure of $\mathcal{S}_{\beta}$ must be $\geq N$. As $N$ was arbitrary, this means that the $H^{2}$-closure of $\mathcal{S}_{\beta}$ must have infinite codimension in $H^{2}$.

\section{References}

[1] Baladi, V., Positive transfer operators and decay of correlations. Advanced Series in Nonlinear Dynamics, 16. World Scientific Publishing Co., Inc., River Edge, NJ, 2000.

[2] Benedicks, M., On Fourier transforms of functions supported on sets of finite Lebesgue measure. J. Math. Anal. Appl. 106 (1985), no. 1, 180-183.

[3] Blank, M., Discreteness and continuity in problems of chaotic dynamics. Translations of Mathematical Monographs, 161. American Mathematical Society, Providence, RI, 1997.

[4] Blasi-Babot, D., Heisenberg uniqueness pairs for three parallel lines. Proc. Amer. Math. Soc., to appear.

[5] Boyarsky, A., Góra, P., Laws of chaos. Invariant measures and dynamical systems in one dimension. Probability and its Applications. Birkhäuser Boston, 1997.

[6] Bugiel, P., A note on invariant measures for Markov maps of an interval. Z. Wahrsch. Verw. Gebiete 70 (1985), no. 3, 345-349.

[7] Chakraborty, S., Rao, B. V., $\theta$-expansions and the generalized Gauss map. Probability, statistics and their applications: papers in honor of Rabi Bhattacharya, 49-64, IMS Lecture Notes Monogr. Ser., 41, Inst. Math. Statist., Beachwood, OH, 2003.

[8] Cornfeld, I. P.; Fomin, S. V.; Sinai, Ya. G., Ergodic theory. Translated from the Russian by A. B. Sosinskii. Grundlehren der Mathematischen Wissenschaften [Fundamental Principles of Mathematical Sciences], 245. Springer-Verlag, New York, 1982.

[9] Driebe, D., Fully chaotic maps and broken time symmetry. Nonlinear Phenomena and Complex Systems, 4. Kluwer Academic Publishers, Dordrecht, 1999.

[10] Hardy, G. H., A theorem concerning Fourier transforms. J. London Math. Soc. 8 (1933), 227-231.

[11] Havin, V., Jöricke, B., The uncertainty principle in harmonic analysis. Ergebnisse der Mathematik und ihrer Grenzgebiete (3) [Results in Mathematics and Related Areas (3)], 28. Springer-Verlag, Berlin, 1994.

[12] Hedenmalm, H., Montes-Rodríguez, A., Heisenberg uniquenes pairs and the Klein-Gordon equation. Ann. of Math. (2) 173 (2011), 1507-1527.

[13] Hedenmalm, H., Montes-Rodríguez, A., Fourier uniqueness sets and the Klein-Gordon equation. Submitted.

[14] Hofbauer, F., Keller, G., Ergodic properties of invariant measures for piecewise monotonic transformations. Math. Z. 180 (1982), 119-140.

[15] Ionescu-Tulcea, C. T., Marinescu, G., Théorie ergodique pour des classes d'opérations non complètement continues. Ann. of Math. (2) 52 (1950), 140-147.

[16] Iosifescu, M, Grigorescu, Ş., Dependence with complete connections and its applications. Cambridge Tracts in Mathematics, 96. Cambridge University Press, Cambridge, 1990.

[17] Kraaikamp, C., Lopes, A. The theta group and the continued fraction expansion with even partial quotients. Geom. Dedicata 59 (1996), no. 3, 293-333.

[18] Keller, G., On the rate of convergence to equilibrium in one-dimensional systems. Comm. Math. Phys. 96 (1984), 181-193.

[19] Lasota A., Yorke, J. A., Existence of invariant measures for piecewise monotonic transformations. Trans. Amer. Math. Soc. 186 (1973), 481-488.

[20] Lev, N., Uniqueness theorems for Fourier transforms. Bull. Sci. Math. 135 (2011), 134-140. 
[21] Matheson, A. L., Stessin, M. I., Cauchy transforms of characteristic functions and algebras generated by inner functions. Proc. Amer. Math. Soc. 133 (2005), 3361-3370.

[22] Pollicott, M., Yuri, M., Dynamical systems and ergodic theory. London Mathematical Society Student Texts, 40. Cambridge University Press, Cambridge, 1998.

[23] Salem, R. On some singular monotonic functions which are strictly increasing. Trans. Amer. Math. Soc. 53 (1943), 427-439.

[24] Schaefer, H. H., Banach lattices and positive operators. Grundlehren der mathematischen Wissenschaften [Fundamental Principles of Mathematical Sciences], 215. Springer-Verlag, New YorkHeidelberg, 1974.

[25] Sjölin, P., Heisenberg uniqueness pairs and a theorem of Beurling and Malliavin. Bull. Sci. Math. 135 (2011), 123-133.

[26] Vallée, B., Dynamical analysis of a class of Euclidean Algorithms. Available at: http://users.info.unicaen.fr/ brigitte/Publications/latinlong5.ps

Canto-Martín: Department of Mathematical Analysis, University of Sevilla, Sevilla, SPAIN E-mail address: fcanto@us.es

Hedenmalm: Department of Mathematics, The Royal Institute of Technology, S - 10044 Stockholm, SWEDEN

E-mail address: haakanh@math.kth.se

Montes-Rodríguez: Department of Mathematical Analysis, University of Sevilla, Sevilla, SPAIN

E-mail address: amontes@us.es 